\title{
Nitric Oxide Acts as a Volume Transmitter to Modulate Electrical Properties of Spontaneously Firing Neurons via Apamin-Sensitive Potassium Channels
}

\author{
Liana Artinian, Karine Tornieri, Lei Zhong, Deborah Baro, and Vincent Rehder \\ Department of Biology, Georgia State University, Atlanta, Georgia 30302-4010
}

\begin{abstract}
Nitric oxide (NO) is a radical and a gas, properties that allow NO to diffuse through membranes and potentially enable it to function as a "volume messenger." This study had two goals: first, to investigate the mechanisms by which NO functions as a modulator of neuronal excitability, and second, to compare NO effects produced by NO release from chemical NO donors with those elicited by physiological NO release from single neurons. We demonstrate that NO depolarizes the membrane potential of B5 neurons of the mollusk Helisoma trivolvis, initially increasing their firing rate and later causing neuronal silencing. Both effects of NO were mediated by inhibition of Ca-activated iberiotoxin- and apamin-sensitive $\mathrm{K}$ channels, but only inhibition of apamin-sensitive $\mathrm{K}$ channels fully mimicked all effects of NO on firing activity, suggesting that the majority of electrical effects of $\mathrm{NO}$ are mediated via inhibition of apamin-sensitive $\mathrm{K}$ channels. We further show that single neurons release sufficient amounts of NO to affect the electrical activity of B5 neurons located nearby. These effects are similar to NO release from the chemical NO donor NOC-7 [3-(2-hydroxy-1-methyl-2-nitrosohydazino)-N-methyl-1propyanamine], validating the use of NO donors in studies of neuronal excitability. Together with previous findings demonstrating a role for NO in neurite outgrowth and growth cone motility, the results suggest that NO has the potential to shape the development of the nervous system by modulating both electrical activity and neurite outgrowth in neurons located in the vicinity of NO-producing cells, supporting the notion of $\mathrm{NO}$ functioning as a volume messenger.
\end{abstract}

\section{Introduction}

Nitric oxide (NO) is involved in many processes in the nervous system, including cell proliferation, neuronal development, and learning and memory (Contestabile and Ciani, 2004; Bicker, 2005; Garthwaite, 2008). One mechanism by which NO affects neuronal function is by modulating electrophysiological properties of neurons (Koh and Jacklet, 2001; Clasadonte et al., 2008; Yang and Cox, 2008), although the physiological relevance and mechanisms of this modulation remain to be determined. NO can reset the level of neuronal activity and excitability by affecting specific ion channels, which, in turn, change the firing rate, the threshold of action potentials (APs), and the ability of neurons to respond to excitatory and inhibitory signals (Ahern et al., 2002; Frick and Johnston, 2005; Steinert et al., 2008). Whereas the role of neuronal activity in determining and modulating synaptic connections is well established in the adult nervous system (Sjöström et al., 2008), its role in developing or regenerating neurons is less understood (Spitzer, 2006). Although long-lasting spiking activity inhibits axonal outgrowth and causes growth cone collapse in invertebrate and vertebrate neurons (Cohan and Kater,

\footnotetext{
Received Sept. 5, 2009; revised Nov. 2, 2009; accepted Dec. 8, 2009.

This work was supported by National Science Foundation Grants 0343096 and 0843173 (V.R.) and stipends from the Brains and Behavior Area of Focus (Georgia State University) (K.T., L.Z.).

Correspondence should be addressed to Dr. Vincent Rehder, Department of Biology and Neuroscience Institute,

Georgia State University, P.0. Box 4010, Atlanta, GA 30302-4010. E-mail: vrehder@gsu.edu.

K. Tornieri's present address: Department of Cell Biology, Emory University, Atlanta, GA 30322.

D0I:10.1523/JNEUROSCI.4511-09.2010

Copyright $\odot 2010$ the authors $\quad 0270-6474 / 10 / 301699-13 \$ 15.00 / 0$
}

1986; Fields et al., 1990), transient bursts of electrical activity can stall growth cone motility, mimicking neurotransmitter-induced firing of APs and growth cone stalling (Ibarretxe et al., 2007).

We showed previously that $\mathrm{NO}$ affects growth cones of identified neurons of the snail Helisoma trivolvis by stimulating a rapid and transient increase in filopodia length and a concentration-dependent slowing in neurite outgrowth (Van Wagenen and Rehder, 1999; Van Wagenen et al., 1999; Trimm and Rehder, 2004; Welshhans and Rehder, 2005). These effects of NO were mediated by $\mathrm{Ca}$ release from $\mathrm{Ca}$ stores and by $\mathrm{Ca}$ entry via voltage-activated Ca channels (Welshhans and Rehder 2007). Because neuronal $\mathrm{Ca}$ transients are markers of change in neuronal excitability (Spitzer et al., 2000), we hypothesized that NOmediated effects on growth cone behavior could be, at least partially, mediated by modulation of electrical properties.

We found that $\mathrm{NO}$ initially increased the neuronal firing rate in B5 neurons and later resulted in long-lasting silencing. These effects were fully mimicked by inhibition of apamin-sensitive (SK-like) K channels and, partially, by inhibition of iberiotoxin (IBTX)-sensitive (BK-like) $\mathrm{K}$ channels, suggesting that $\mathrm{Ca}$ activated apamin-sensitive SK-type channels were the main target of NO in modulating the electrical activity of B5 neurons. This is the first example in which NO is shown to exert a long-lasting decrease in neuronal excitability via inhibition of apaminsensitive $\mathrm{K}$ channels.

$\mathrm{NO}$ donors are convenient tools to produce $\mathrm{NO}$ and are used widely to investigate physiological effects of NO. Studies using NO release from neurons as a means of NO delivery, however, are 
considered more physiologically relevant. Here we compared the effects of the chemical NO donor NOC-7 [3-(2-hydroxy-1methyl-2-nitrosohydazino)- $N$-methyl-1-propyanamine] with $\mathrm{NO}$ release from a single neuron and demonstrated for the first time that NO from both sources modulated neuronal intrinsic electrical properties in a similar manner. Our results support the notion that cellular NO release can modulate neuronal firing properties in synaptically unconnected neurons, in direct support of NO functioning as a volume messenger.

\section{Materials and Methods}

Neuronal culture. Identified B5 and B19 neurons were dissected from $H$. trivolvis buccal ganglia and plated for neuronal culture. Neurons were plated on poly-L-lysine (hydrobromide; molecular weight of 70,000150,$000 ; 0.25 \mathrm{mg} / \mathrm{ml}$; Sigma) coated glass coverslips glued to the bottom of $35 \mathrm{~mm}$ cell culture dishes (Falcon 1008). Cells were grown overnight at room temperature and used for electrophysiological recordings $24-48 \mathrm{~h}$ after plating. Cells were plated into conditioned medium (CM), prepared by incubating snail brains in Leibowitz L-15 medium (Invitrogen) for 3-4 d. The composition of L-15 medium was as follows: $44.6 \mathrm{~mm} \mathrm{NaCl}$, $1.7 \mathrm{~mm} \mathrm{KCl}, 1.5 \mathrm{~mm} \mathrm{MgCl}_{2}, 0.3 \mathrm{~mm} \mathrm{MgSO}_{4}, 0.14 \mathrm{~mm} \mathrm{KH}_{2} \mathrm{PO}_{4}, 0.4 \mathrm{~mm}$ $\mathrm{Na}_{2} \mathrm{HPO}_{4}, 1.6 \mathrm{~mm}$ Na pyruvate, $4.1 \mathrm{~mm} \mathrm{CaCl}, 5 \mathrm{~mm} \mathrm{HEPES}, 50 \mu \mathrm{g} / \mathrm{ml}$ gentamicin, and $0.15 \mathrm{mg} / \mathrm{ml}$ glutamate in distilled water, $\mathrm{pH} 7.4$. As described previously (Tornieri and Rehder, 2007), neurons referred to as "senders" were placed into hemolymph-coated glass dishes according to Spencer et al. (2000) and kept at room temperature for $24 \mathrm{~h}$. At time of experimentation, they were transferred to an experimental dish containing neurons to be tested for their response to $\mathrm{NO}$ and maneuvered into close proximity of the cell body of receiver neurons with the help of a poly-lysine-coated microelectrode.

Electrophysiology. Cultured neurons grown in $35 \mathrm{~mm}$ dishes were placed on a fixed platform located above an inverted microscope (Sedival) that was mounted on a manual translator (Siskiyou). This configuration permitted us to move the field of view after establishing whole-cell configuration. Low-resistance microelectrodes (3-4 M $\Omega$ ) were pulled from borosilicate glass tube (outer diameter, $1.5 \mathrm{~mm}$; inner diameter, $0.86 \mathrm{~mm}$; Sutter Instruments) on a Sutter Instruments micropipette puller (P-87) and heat-polished (Micro Forge MF-830; Narishige). Neurons were recorded in cell-attached and whole-cell configuration using a patch-clamp amplifier (Axon 200B; MDS Analytical Technologies) and an analog-to-digital converter (Digidata 1380 system). Data acquisition and analysis were performed using MDS Analytical Technologies pClamp software (version 10). Current-clamp configuration was used to record membrane potential, firing activity, and AP characteristics, as well as to evoke depolarizing or hyperpolarizing currents. Voltage-clamp configuration was used to evaluate whole-cell configuration parameters and to generate voltage-current protocols (holding voltage of $-60 \mathrm{mV}$ ). Leibowitz L-15 medium (Invitrogen) was used normally as extracellular solution. In some experiments, L-15 medium was replaced with: (1) normal saline containing extracellular solution [in mu: $51.3 \mathrm{NaCl}, 1.7$ $\mathrm{KCl}, 4.1 \mathrm{CaCl}_{2}, 1.5 \mathrm{MgCl}_{2}$, and 5 HEPES, pH 7.3 (127 mOsm)] or (2) $\mathrm{NaCl}$-free solution [in mM: $51.3 \mathrm{~N}$-methyl-D-glucamine (NMDG), 1.7 $\mathrm{KCl}, 4.1 \mathrm{CaCl}_{2}, 1.5 \mathrm{MgCl}_{2}$, and 5 HEPES, pH 7.3 (127 mOsm)]. Intracellular recording solution contained the following (in $\mathrm{mm}$ ): 54.4 K-aspartate, $100 \mu \mathrm{M}$ EGTA, $2 \mathrm{MgCl}_{2}, 5$ HEPES, 5 dextrose, and 5 ATP.

Recordings were filtered at $5 \mathrm{kHz}(-3 \mathrm{~dB}$, four-pole Bessel filters). Immediately after obtaining the whole-cell configuration, the resting membrane potential was recorded from the amplifier panel meter. The input resistance was determined from the membrane test function (pClamp 10) by giving a $+5 \mathrm{mV}$ voltage pulse. In voltage-clamp experiments, the input resistance was monitored for stability of the whole-cell configuration, and experiments were excluded from analysis if input resistance changed $>10 \%$. AP kinetics and frequency were analyzed using the "Event Detection" function (pClamp, Clampfit 10, Template Search option). The amplitude of afterhyperpolarization potentials (AHPs) elicited by depolarizing current injection (1 nA for $1 \mathrm{~s}$ ) was measured $500 \mathrm{~ms}$ after the end of current injection. The AHP amplitude of single rebound APs was measured 200 or $300 \mathrm{~ms}$ after repolarization of the membrane potential after the AP and is expressed as a percentage change between control and experimental conditions. AP width at half-amplitude was measured for the second AP evoked after stimulation. The significance of the effects on above parameters was evaluated with one-way ANOVA analysis and Tukey's post hoc test, using Origin 6 software. Significant differences are indicated as ${ }^{\star} p \leq 0.05,{ }^{* *} p \leq 0.01$, and ${ }^{* * *} p \leq 0.001$.

Molecular cloning of $\mathrm{H}$. trivolvis NO synthase and double-stranded RNA synthesis. Neuronal nitric oxide synthase (NOS) is highly conserved across species. Degenerative primers were designed after cDNA analysis of Aplysia californica, Lymnaea stagnalis, Gecarcinus lateralis (Bermuda land crab), Drosophila melanogaster, and mouse neuronal NOS. We cloned Helisoma trivolvis NOS (HeliNOS) from snail brains and buccal ganglia by reverse transcription-PCR using degenerate primers corresponding to a conserved region between the heme-binding and the calmodulin-binding recognition sites. Total RNA was extracted from 30 H. trivolvis brains and 30 buccal ganglia using Qiagen RNA Easy Mini kit. SuperScript II Reverse Transcriptase (Invitrogen) was used to generate cDNA from a total of $2.75 \mu \mathrm{g}$ of RNA at $50^{\circ} \mathrm{C}$ for $2 \mathrm{~h}$. The first PCR (40 cycles at $94^{\circ} \mathrm{C}$ for $1 \mathrm{~min}, 50^{\circ} \mathrm{C}$ for $1 \mathrm{~min}, 72^{\circ} \mathrm{C}$ for $2 \mathrm{~min}$ ) was performed using the forward primer $1 \mathrm{~L} 5^{\prime}$-CARTGGWSIAARYTICARGTITTYGAYGC- $3^{\prime}$ and the reverse primer $2 \mathrm{R} 5^{\prime}$ - ACCCAICCISNIGGRCANCCNCC- $3^{\prime}$, and the product of this reaction was used for the nested PCRs using the forward primer $1 \mathrm{~L}$ and the reverse primer $1 \mathrm{R} 5^{\prime}$ CCANCCRTTRAAIGGRCAISIIGGRAAYTC-3' (40 cycles at $94^{\circ} \mathrm{C}$ for 1 $\min , 55^{\circ} \mathrm{C}$ for $1 \mathrm{~min}, 72^{\circ} \mathrm{C}$ for $2 \mathrm{~min}$ ) and the forward primer $2 \mathrm{~L} 5^{\prime}$ GGNGAYCCIGCIAAYGTIGARTTYACNGA- $3^{\prime}$ and the reverse primer $2 \mathrm{R}$ ( 40 cycles at $94^{\circ} \mathrm{C}$ for $1 \mathrm{~min}, 55^{\circ} \mathrm{C}$ for $1 \mathrm{~min}, 72^{\circ} \mathrm{C}$ for $2 \mathrm{~min}$ ). Identified segments $1 \mathrm{~L}-1 \mathrm{R}$ and $2 \mathrm{~L}-2 \mathrm{R}$ were cloned into the pcR2.1 vector with T7 promoter (Invitrogen) and sequenced (Georgia State University DNA Sequencing Facility, Atlanta, GA). Data were analyzed with DNASTAR and CLC Free Workbench 3.2.2 (CLC Bio), and submitted to GenBank under accession number GQ178288.

A total of 240 amino acids encoded by 720 bp of the identified overlapping sequences and their alignment with highly homologous sequences from the listed above species are shown in Figure $1 \mathrm{~A}$. HeliNOS had $95 \%$ identity to the corresponding segment of apNOS from A. californica and demonstrated high similarity with the corresponding segment of mammalian neuronal NOS. Figure $1 B$ shows a schematic representation of mammalian neuronal NOS with its conserved regulatory sites. The cloned sequence is located between the highly conserved heme- and calmodulin-binding sites.

To produce double-stranded RNA (dsRNA), the 523 bp segment $1 \mathrm{~L}-1 \mathrm{R}$ was cloned into the pcR2.1 vector in forward and reverse directions, and these two constructs were used to generate sense and antisense RNAs using T7 MEGAscript kit (Ambion). Each strand was purified using Purification System and NucAway Spin Columns (Ambion). The dsRNA was generated by annealing equimolar amounts of sense and antisense transcripts $\left(90^{\circ} \mathrm{C}\right.$ for $15 \mathrm{~min}$ and then overnight at room temperature) and subsequently aliquoted and stored at $-20^{\circ} \mathrm{C}$. To knockdown HeliNOS in cultured B5 neurons, dsRNA interference (dsRNAi) was added to cell culture dishes at a concentration of $1 \mu \mathrm{g} / \mathrm{ml}$ for $24 \mathrm{~h}$.

Measurement of growth cone dynamics. Growth cones were viewed under a $100 \times$ oil-immersion objective on a Sedival microscope. A regular CCD camera (C-72; Dage-MTI) was used to capture phase-contrast images, which were digitized on a frame grabber (Scion LG-3; Scanalytics) and analyzed with Scion Image software (Scion Corporation) on a Hewlett-Packard Pavilion. Images were captured before ( -5 and $0 \mathrm{~min})$ and at defined times $(2,5,10,15,20$, and $30 \mathrm{~min})$ after maneuvering a sender cell body into the vicinity of a growth cone. Filopodial length was analyzed by measuring the length of all filopodia on a growth cone from their tip to the edge of the central domain, thereby preventing potential incorrect measurements influenced by lamellipodial dynamics. Filopodial length changes are expressed as a percentage change normalized to the time point $t=0$ just before the addition of a sender. ANOVA (repeated-measures ANOVA with time as repeated measures when appropriate) was performed with SuperANOVA (Abacus Concepts) to determine statistical significance between conditions. Fisher's protected least significant difference test was used as post hoc analysis of preplanned 
A

AAK83069 Aplysia californica AAW88577 L.stagnalis NP 032738 Mus musculus AAT46681 Gecarcinus lateralis AAF25682 Drosophila melanogaster NOS Helisoma Trivolvis

AAK83069 Aplysia californica AAW88577 L.stagnalis

NP_032738 Mus musculus AAT46681 Gecarcinus lateralis AAF25682 Drosophila melanogaster NOS Helisoma Trivolvis

AAK83069 Aplysia californic AAW88577 L.stagnalis

NP_032738 Mus musculus

AAT46681 Gecarcinus lateral

AAF25682 Drosophila melanogaster NOS Helisoma Trivolvis

AAK83069 Aplysia californic AAW88577 L.stagnalis

NP 032738 Mus musculus

AAT46681 Gecarcinus lateralis AAF25682 Drosophila melanogaster NOS Helisoma Trivolvis

AK83069 Aplysia californica AAW88577 L.stagnalis

NP_032738 Mus musculus

AAT46681 Gecarcinus lateralis $K$ AAF25682 Drosophila melanogaster NOS Helisoma Trivolvis

$1 \mathrm{~L}$

QWSKLQVFDA RHILTARGMY QWSKLQVFDA REIETPREM QWSKLQVFDA RDCTTAHGM

QWSKLQVFDA RYVSTASGMF QWSKLQVFDC RYVTTTSGM

......... RHILTARGTY

$$
8021
$$

$$
80 \text { 2L }
$$

IRYAGYKMED GSIVGDPANV EFTEQCVKLG WKPKYGMFD

LPLVLSAAEN SPEYFELPTE 120

IRYAGYKQPD GSTLGDPANV EFTEICIQQG WKPPRGRFDV LPLLLQANGN DPELFQIPPE 120

I SYAGYKQED GS IVGDPLNV EFTEVCQRLG WRGKGGRWDV LPLVLSASGH DPEWFDIPPE 120 ISYAGYKQAD GKIIGDPMNV EFTEVCTKLG WKSKGSEWD I LPLVVSANGH DPDYFDYPPE 120 IRYAGYKMDD GKVIGDPANV EFTDQCIKLG WKPKYGMFDV LPLVLSAAGS DPEWFEIPPE 110 140

LVLEVNMKHP KHPWFADLG LVLEVTLKHP EYPWFAEMG 160 1R 180

KWYALPAVSG MLFDCGGLEF PACPFNGWYM GTEIGARDFC 180 WYALPSG MLLDCGGLEF PSCPFNGWFM GTMIGSRNLC 180 IILTVPITHP EYKWFQELDL QWYGLPGVSS LMFDCGGLEF PAAPFNGWYM VSEIGTRDLC 180 LILEVPLTHP KFEWFSDLGL RWYALPAVSS MLFDVGGIQF TATTFSGWYM STEIGSRNLC 180 LILEVNIRHP KYPWFADMGL KWYALPAVSG MLFDCGGLEF PCCPFNGWYM GTEIGARDFC 170 200 220 240

I

DANRYNMMET VATKMGLDTR KSASLWKDRV LVEVNVAVLY SFQTSGVTIT DHHAASESFI 240 DPHRYNMLEP IGLKMGLNTE TASSLWKDQV LIEANVAVLF SFESANVTIV NHHDASTDFI 240 DNSRYN ILEE VAKKMDLDMR KTSSLWKDQA LVEINIAVLY SFQSDKVTIV DHHSATESFI 240 DPHRYNILET VGRRMGLDTR SPTNLWKDKA LVEVNIAVLH SFQSLNVTIV DHHSAAESFM 240 DTNRRNMLET VALKMQLDTR TPTSLWKDKA VVEMNIAVLH SYQSRNVTIV DHHTASESFM 240 DANRYNLLEP IATRMGLDTK KSSSLWKDRA LVEINLAVLY SFQTSGVTIT DHHAASESFI 230 2R

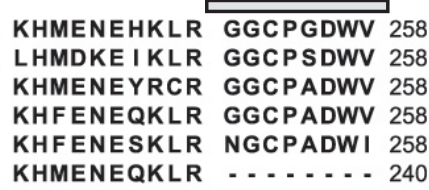

B

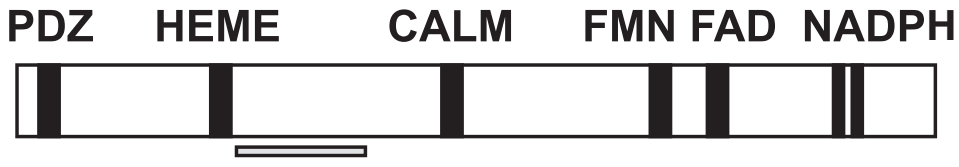

Figure 1. Partial cloning of HeliNOS, RNAi design, and sequence alignment of $H$. trivolvis neuronal NOS with other known NOS isoforms. $A$, The 240 aa (720 bp cDNA segment) of $H$. trivolvis NOS is aligned with homologous regions of NOS from A. californica, L. stagnalis, mouse, G. lateralis (Bermuda land crab), and D. melanogaster. The boxed areas indicate the location of primer alignment. Black bar denotes the segment used for RNAi production. B, Schematic of mammalian NOS indicating a series of conserved binding domains such as PDZ domain, heme, calmodulin (CALM), flavin mononucleotide (FMN), flavin adenine dinucleotide (FAD), and NADPH binding domains. The black bar below indicates the location of the segment cloned from H. trivolvis NOS; the white bar denotes the sequence used to make RNAi.

comparisons. Significant differences are indicated as ${ }^{*} p \leq 0.05,{ }^{* *} p \leq$ 0.01 , and ${ }^{* * *} p \leq 0.001$.

Immunocytochemistry. Growth cones were fixed and treated according to Cohan et al. (2001). Neurons were fixed in $0.1 \%$ glutaraldehyde and $4 \%$ paraformaldehyde (in Small's buffer; Electron Microscopy Sciences) for $5 \mathrm{~min}$, washed twice with Small's buffer, lysed in $0.1 \%$ Triton X-100 (Sigma) for $5 \mathrm{~min}$, washed twice with Small's buffer, incubated in $100 \mathrm{~mm}$ glycine for $10 \mathrm{~min}$, washed twice with Small's buffer, and then blocked with Blotto (nonfat instant dry milk, 0.05\% Tween 20 in PBS) for $30 \mathrm{~min}$. Next they were incubated with a polyclonal rabbit anti-neuronal NOS (nNOS) antibody (Affinity Bioreagents) at a dilution of 1:1000 for $1 \mathrm{~h}$ at $37^{\circ}$, washed three times in PBS-BSA buffer (PBS and 0.05\% BSA), incubated with a secondary antibody, Alexa Fluor 488 goat anti-rabbit (Invitrogen), at a dilution of 1:300 for $30 \mathrm{~min}$ at $37^{\circ}$, and finally washed twice in PBS-BSA buffer before mounting. Growth cones were viewed through a $100 \times$ oil-immersion objective on a Zeiss Axiovert inverted microscope equipped with an FITC filter set. Images were acquired using a cooled Photometrics CCD camera and stored on a Macintosh computer (Apple Computers). A camera exposure time of $100 \mathrm{~ms}$ was used for all images. Fluorescent intensity was measured with Scion Image software (Scion Corporation) by drawing a box over individual growth cones and averaging the signal contained within. Staining was done in parallel for different experimental conditions to minimize differences in staining intensity between dishes. Statistical analysis of the fluorescence intensity was performed by using a $t$ test for independent samples with SuperANOVA (Abacus Concepts). Significant differences are indicated as ${ }^{*} p \leq$ $0.05,{ }^{* *} p \leq 0.01$, and ${ }^{* * *} p \leq 0.001$.

Solutions and drugs. The nitric oxide donor NOC-7 (Calbiochem) was dissolved in $100 \mathrm{~mm} \mathrm{NaOH}$ to make a stable stock solution of $100 \mathrm{~mm}$ NOC-7. NOC-7 ( $2 \mu \mathrm{l}$ of stock solution) was mixed with $200 \mu \mathrm{l}$ of extracellular solution removed from the recording dish and immediately added back into the dish to give a final concentration of $100 \mu \mathrm{M}$ NOC-7. $\mathrm{NaOH}$ at $100 \mu \mathrm{m}$ did not change the $\mathrm{pH}$ of the solution and did not affect the firing rate or the membrane potential of $\mathrm{B} 5$ neurons.

Other drugs used included NMDG as Na replacement, the Ca channel blockers $\mathrm{CdCl}_{2}$ and $\mathrm{LaCl}_{3}$, the BK channel blocker IBTX (Sigma), and the SK channel blocker apamin (Alomone Labs). Water-insoluble agents were dissolved in dimethylsulfoxide (Sigma).

\section{Results}

Initial characterization of basic membrane properties and electrical activity of $\mathrm{B} 5$ neurons in cell culture

To address how NO affected electrical activity and excitability of B5 neurons, we first analyzed their basic electrical properties. All experiments were performed on neurons that had been in culture 
A

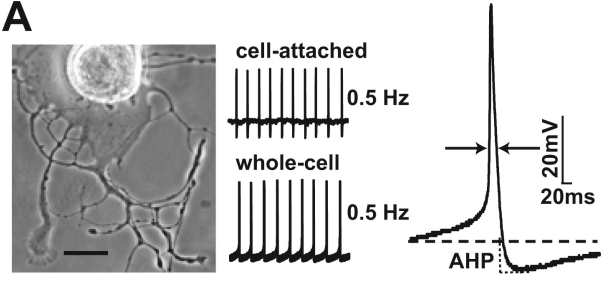

B
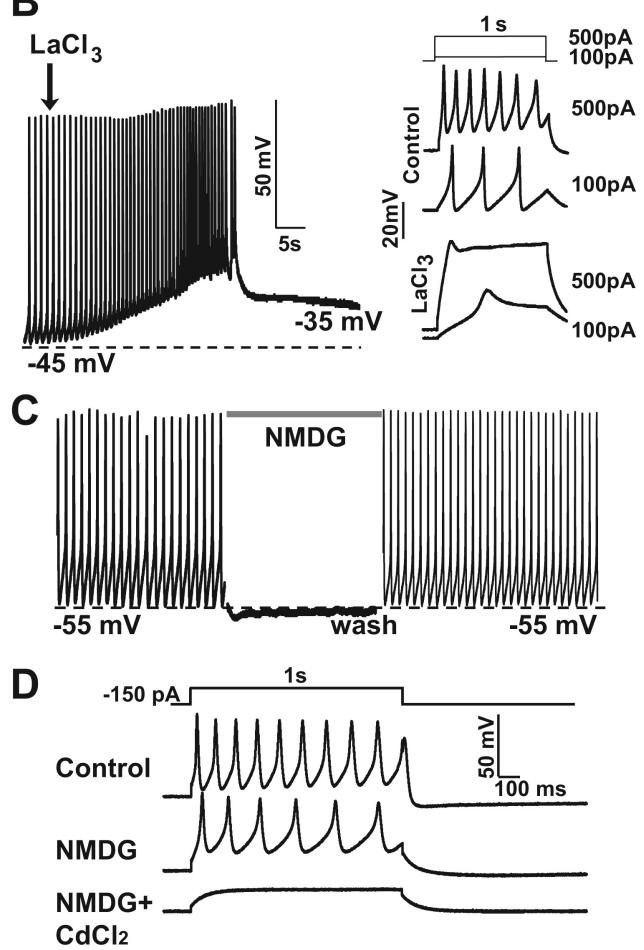

Figure 2. Basic membrane properties and electrical activity of the B5 neurons in culture. $\boldsymbol{A}$, Left, Example of a mature B5 neuron with well developed neurites and a large growth cone. Scale bar, $50 \mu \mathrm{m}$. Middle, Spontaneous tonic firing activity of a mature B5 neuron recorded in cell-attached (top) and whole-cell current-clamp (bottom) modes. Right, Shape of a typical spontaneous AP, depicting amplitude, width at half-amplitude, and AHP. B, Left, Spontaneous tonic firing activity was blocked after voltage-gated $\mathrm{Ca}$ channels were blocked with $\mathrm{LaCl}_{3}(0.5$ $\mathrm{mm})$. Note that the membrane potential remained elevated in the continued presence of $\mathrm{LaCl}_{3}$, consistent with a closing of (a-activated $\mathrm{K}$ channels. Right, $\mathrm{LaCl}_{3}(0.5 \mathrm{~mm})$ inhibited evoked APs normally induced by steps of positive current injection (50-500 pA, $\Delta 50$ pA, 1 s). C, Spontaneous tonic firing activity was blocked after Na ions were replaced with NMDG. The effect of NMDG was reversible. $D, B 5$ neurons responded to positive current injection (150 pA, 1s) with firing of evoked APs. After Na ion replacement with NMDG, current injection still elicited Ca-based APs that were blocked by the $\mathrm{Ca}$ channel inhibitor $\mathrm{CdCl}_{2}(1 \mathrm{~mm})$. Note that current injections up to 2 $\mathrm{nA}$ were unable to evoke APs in neurons treated with NMDG and $\mathrm{CdCl}_{2}$ (data not shown).

for 1 or $2 \mathrm{~d}$ and that had well developed neurites with motile growth cones (Fig. $2 \mathrm{~A}$, left). All neurons were grown in isolation so that the recorded activity reflected spontaneous, cell-intrinsic electrical properties. B5 neurons had a resting membrane potential of $-43 \pm 7 \mathrm{mV}$ and exhibited a spontaneous tonic firing activity with an average frequency of $0.78 \pm 0.43 \mathrm{~Hz}(n=57)$ that was stable for at least $3 \mathrm{~h}$ of continuous whole-cell recording. To rule out potential side effects of the whole-cell configuration on the spiking activity, we compared spiking rates before breaking through the cell membrane (in cell-attached configuration) and after establishing the whole-cell configuration. The frequencies of spontaneous tonic spiking activity in the cell-attached and whole-cell configuration recorded from the same neurons remained basically unchanged $(n=3)$ (Fig. $2 A$, middle), indicating no obvious adverse effect of the whole-cell configuration on spik- ing activity. The typical spontaneous was characterized by an amplitude of $76 \pm 9 \mathrm{mV}$ and a half-width of $18 \pm 3 \mathrm{~ms}(n=5)$, followed by a prolonged AHP (Fig. $2 \mathrm{~A}$, right).

Ca channels are main contributors to APs in gastropods (Adams et al., 1980). To investigate the ionic basis of the AP in B5 neurons, we used inhibitors of voltage-gated Ca channels, $\mathrm{LaCl}_{3}$ and $\mathrm{CdCl}_{2}$, to evaluate the contribution of Ca channels to spontaneous and evoked APs. The blockage of Ca channels with $\mathrm{LaCl}_{3}$ inhibited spontaneous APs within 1 min of application (Fig. $2 B$, left) and also blocked depolarization-evoked APs at all current injections ranging from 50 to $500 \mathrm{pA}$ (Fig. $2 \mathrm{~B}$, right). The same results were obtained with the $\mathrm{Ca}$ channel blocker $\mathrm{CdCl}_{2}$ (data not shown). Together, these data suggest that B5 neurons depend on functional Ca channels to fire spontaneous and evoked APs.

To investigate the contribution of $\mathrm{Na}$ channels to the spontaneous spiking of B5 neurons, we replaced extracellular $\mathrm{Na}$ ions with the impermeable monovalent cation NMDG. Na replacement became necessary, because tetrodotoxin (TTX) up to $50 \mu \mathrm{M}$ did not have an effect on Na channels in B5 neurons (data not shown) and, therefore, could not be used in this study. This finding was consistent with results by others that many gastropod sodium channels are apparently resistant to TTX (Staras et al., 2002). Na replacement with NMDG caused a small hyperpolarization of $-4 \pm 1 \mathrm{mV}(n=12)$ and resulted in the blockade of spontaneous APs, suggesting that $\mathrm{Na}$ currents contribute to the resting membrane potentials and that the spontaneously occurring spikes require an $\mathrm{Na}$ conductance (Fig. 2C). The effect of $\mathrm{Na}$ replacement was reversible, and the spontaneous activity recovered after NMDG was replaced with normal Na-containing solution. Interestingly, in the absence of $\mathrm{Na}$ ions, injection of depolarizing currents still elicited APs (Fig. 2D) but with distinct characteristics. The half-width of evoked APs in the presence of NMDG was increased to $23.3 \pm 2.8 \mathrm{~ms}$ compared with $13.2 \pm 1.4$ $\mathrm{ms}(150 \mathrm{pA}, p \leq 0.001, n=4)$ in normal saline. The activation and inactivation kinetics of the depolarization-evoked APs were much slower in the presence of NMDG: the rise and decay times were $6.7 \pm 0.1$ and $15.2 \pm 1.6 \mathrm{~ms}$, respectively, in the presence of Na versus $9.7 \pm 0.7 \mathrm{~ms}(p \leq 0.01, n=4)$ and $25.7 \pm 1.8 \mathrm{~ms}(p \leq$ $0.001, n=4)$ in the Na-free solution. In the absence of $\mathrm{Na}$ ions, the APs evoked by positive current steps of different magnitude (up to $+2 \mathrm{nA}$ ) were totally blocked by the $\mathrm{Ca}$ channel blocker $\mathrm{CdCl}_{2}$, suggesting that they were carried by $\mathrm{Ca}$ ions. Together, these data suggest that spontaneous APs in B5 neurons are dependent on both $\mathrm{Na}$ and $\mathrm{Ca}$ conductances and that neurons can fire $\mathrm{Ca}$ APs when depolarized in the absence of $\mathrm{Na}$ ions but that they cannot fire $\mathrm{Na}$ APs when depolarized in the absence of $\mathrm{Ca}$ ions.

The NO donor NOC-7 induces a short-term increase in spiking activity, persistent membrane depolarization, and subsequent silencing of spontaneous spiking activity We showed previously that the gaseous messenger NO affects growth cone motility by increasing intracellular $\mathrm{Ca}$ through a combination of Ca release from intracellular stores and Ca influx (Welshhans and Rehder, 2007). Given that spontaneous and evoked APs in B5 neurons depended on Ca channels, we next questioned whether the NO-mediated $\mathrm{Ca}$ influx reported in the previous study could have resulted from NO acting as a modulator of neuronal firing activity. To characterize the effect of NO on the spontaneous firing activity of B5 neurons, we bath applied the NO donor NOC-7 $(100 \mu \mathrm{M})$ [half-life: 5-10 min at physiological pH (Otsuka et al., 1998; Onodera et al., 2000)] and recorded neurons in whole-cell current-clamp configuration. In a first set of experiments, spontaneously firing neurons were held in 

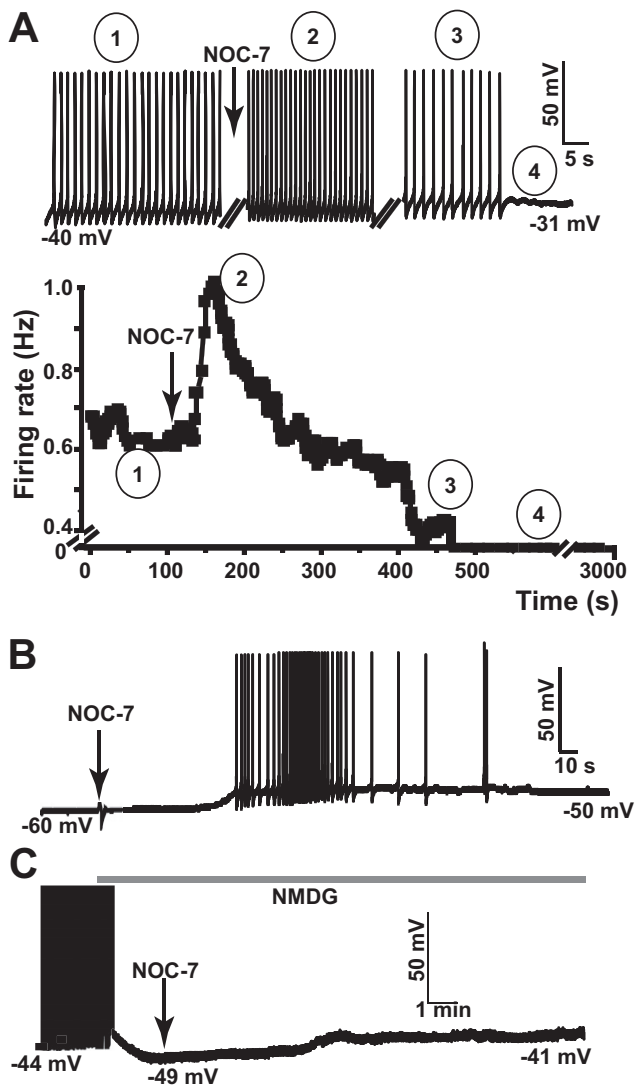

Figure 3. N0 induces a transient increase in spiking activity, membrane depolarization, and subsequent silencing of spontaneous spiking activity. $A$, Top, A spontaneously firing $B 5$ neuron (1) was depolarized and transiently increased its firing rate (2) immediately after treatment with NOC-7 (100 $\mu \mathrm{m}$, bath application; arrow). The spiking frequency subsequently decreased and the neuron stopped firing after $4.5 \mathrm{~min}$ (3). Bottom, Plot of firing rate over time for the neuron shown above, depicting spontaneous firing rate (1), NOC-7- induced transient increase in firing rate (2), slowing of firing activity (3), and subsequent silencing, typically lasting for at least $30 \mathrm{~min}$ (4). Note that circled numbers correspond to time points in the top. $\boldsymbol{B}$, Response of a neuron in current-clamp configuration (injection of negative current to hold $V_{\mathrm{m}}=-60 \mathrm{mV}$ ) to treatment with NOC-7 (100 $\mu \mathrm{m}$, bath application; arrow). The membrane potential of the neuron is depolarized, resulting in an increased firing rate. This initial firing rate increase typically lasted for $<2$ min and was followed by long-lasting silencing of spontaneous firing activity, during which the membrane potential was typically depolarized by $\sim 10 \mathrm{mV}$. C, The replacement of Na with NMDG prevented neuronal firing activity and slightly hyperpolarized the membrane potential. Application of NOC-7 (100 $\mu \mathrm{M})$ depolarized the membrane potential in the absence of extracellular Na ions (NMDG replacement) to the same degree as in the presence of $\mathrm{Na}(\boldsymbol{B})$.

current-clamp mode with 0 current injection. NOC-7 treatment resulted in the depolarization of neurons by $8.5 \pm 1 \mathrm{mV}(n=5)$ and a transient increase in the firing rate lasting $1.5-5 \mathrm{~min}(n=$ 5 ), followed by the total inhibition of spontaneous firing. Figure $3 A$ illustrates an example of a neuron that was originally spiking with $0.63 \pm 0.02 \mathrm{~Hz}$ and that increased its firing rate to $1.12 \pm$ $0.03 \mathrm{~Hz}$ almost immediately after treatment with NOC-7. After $4.5 \mathrm{~min}$ of being depolarized, the neuron stopped firing altogether and remained in this silenced state for at least $30 \mathrm{~min}$ (Fig. $3 A)(n=5)$. Silenced neurons did not respond to a second treatment with NOC-7 (100 $\mu \mathrm{M}, n=4)$, suggesting that the primary effect of NOC-7 on electrical properties of B5 neurons was long lasting and prevented $\mathrm{B} 5$ neurons from responding to an additional stimulation with NO (data not shown).

Because individual neurons exhibited a range of spontaneous spiking rates, we next tested the effect of NOC-7 on neurons hyperpolarized to the same resting membrane potential. To this end, we performed whole-cell current-clamp experiments on B5 neurons and injected a constant negative current $(-15 \pm 5 \mathrm{pA})$ to reach $-60 \mathrm{mV}$, which moved the membrane potential below the threshold for spontaneous firing (Fig. 3B). NOC-7 (100 $\mu \mathrm{M}$, bath application) depolarized the membrane potential and induced bursting behavior within 1-5 min after application of the NO donor. This initial appearance of bursting typically lasted from 1.5 to $2 \mathrm{~min}$ and was followed by a period of at least $30 \mathrm{~min}$, during which neurons were silenced while the membrane potential typically remained depolarized by $9 \pm 1 \mathrm{mV}(n=4)$. Thus, the application of NOC-7 in zero-current condition, when neurons were spontaneously firing, and in hyperpolarizing-current condition, when neurons were silent, had similar outcomes: depolarization of the membrane potential, transient increase in firing activity, and, finally, long-term silencing of spontaneous electrical activity at slightly depolarized membrane potential.

We next investigated the ionic basis of the depolarization induced by NOC-7. When extracellular Na ions were replaced by NMDG, NOC-7 treatment resulted in a depolarization of the same magnitude $(7.5 \pm 1 \mathrm{mV}, n=4)$ as in Na-containing medium, suggesting that the NO-induced depolarization was independent of $\mathrm{Na}$ ions (Fig. 3C). Because an activation of $\mathrm{Ca}$ conductances was unlikely at these negative membrane potentials, it raised the possibility that the NO-induced depolarization at hyperpolarized membrane potentials was mediated by the inhibition of one or several $\mathrm{K}$ conductances.

\section{NO increases the width of evoked APs and decreases AHPs}

To address how NOC-7 might have brought about the depolarization, transient increase in firing rate, and the subsequent silencing, we investigated some key electrophysiological parameters changed by NO, namely AP shape and the AHP following it. Evoked APs elicited by depolarizing current injections (50 pA) before NOC-7 treatment (control) were compared with APs evoked in neurons after they had been silenced (20-30 min after treatment with NOC-7). NOC-7 treatment broadened the width of evoked APs by $15 \pm 3 \%(p \leq 0.01, n=5)$ (Fig. $4 A$, left).

To investigate the effect of NOC-7 on the AHP, we compared the amplitude of AHPs of rebound APs elicited after the return from a small hyperpolarizing current injection $(-0.5 \mathrm{nA}, 1 \mathrm{~s})$. We found that NOC-7 treatment decreased the AHP of rebound APs (Fig. $4 A$, right), a modulation that would result in an increase in neuronal excitability. To investigate the effect of NOC-7 on the AHP in more detail, we used a prolonged depolarizing current injection $(0.5 \mathrm{nA}, 1 \mathrm{~s})$ that resulted in a large AHP. The evoked AHP under control conditions had an amplitude of $9.4 \pm 2.1 \mathrm{mV}$ and was significantly reduced by $85 \pm 12 \%$, to $1.4 \pm 1.1 \mathrm{mV}$ after NOC-7 treatment $(n=5, p \leq 0.001)$ (Fig. $4 B)$. The NOC-7induced spike broadening and inhibition of AHP were consistent with the idea that $\mathrm{NO}$ inhibited one or more K conductances. A closure of K conductances by NO could explain the initial depolarization and increase in firing rate seen after treatment with NOC-7 and would be consistent with an increase in neuronal excitability.

NOC-7 partially inhibits a steady-state outward conductance We next performed voltage-clamp experiments to directly investigate the effect of NOC-7 on outward conductances in B5 neurons. Currents were evoked by $1 \mathrm{~s}$ voltage steps from a holding potential of $-60 \mathrm{mV}$ to test potentials ranging from -100 to +40 $\mathrm{mV}$ in $10 \mathrm{mV}$ increments (Fig. 5A). Sustained outward currents were measured at $980 \mathrm{~ms}$ (arrows) after the onset of each voltage 


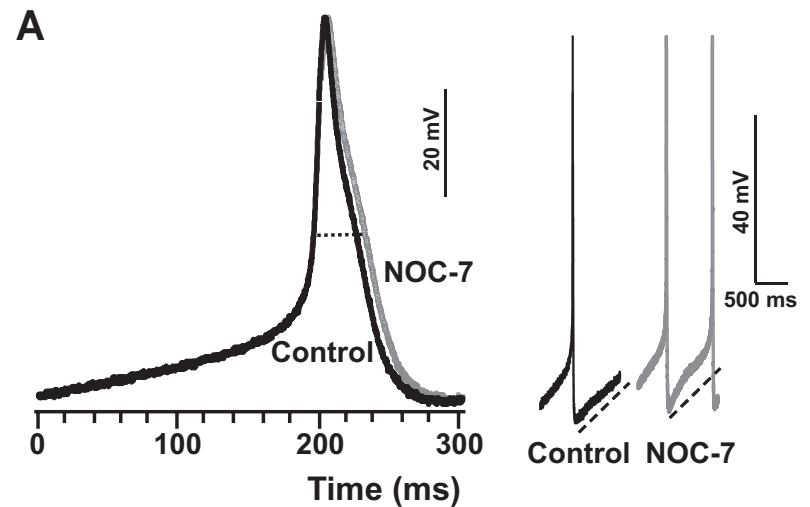

B

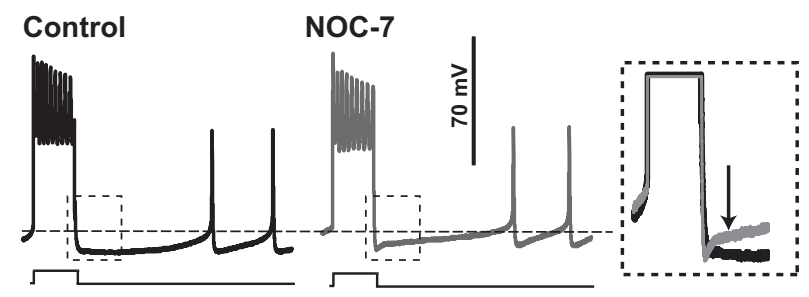

Figure 4. NO broadens evoked APs and attenuates AHP. A, Left, APs (second AP in each train of APs was used for analysis) induced by $50 \mathrm{pA}$ current injection before (black) and after (gray) NOC-7 treatment (100 $\mu \mathrm{m})$. Right, NOC-7 treatment reduced the AHP after rebound APs elicited by small hyperpolarizing current injection ( $-0.5 \mathrm{nA}, 1 \mathrm{~s})$. $B$, NOC -7 treatment attenuated the AHP that was evoked by a depolarization step ( $500 \mathrm{pA}, 1 \mathrm{~s})$. Inset, 0verlap of boxed regions on right show magnification of AHPs under control (black) and NOC-7 treatment (gray) conditions. AHPs were measured $500 \mathrm{~ms}$ after the end of the depolarization step (arrow). NOC-7 treatment inhibited the amplitude of the AHP by $85 \pm 12 \%$ compared with controls $(n=5, p \leq 0.001)$.

step. NOC-7 attenuated the sustained outward current induced by positive voltages. At $+40 \mathrm{mV}$, NOC-7 decreased the outward current to $75 \pm 5 \%$ of control levels ( $p \leq 0.001, n=4)$ (Fig. $5 B, C)$. The inhibitor of Ca-activated or voltage-activated chloride currents, NPPB [5-nitro-2-(3-phenylpropylamino) benzoic acid] $(50 \mu \mathrm{M})$, did not affect the steady-state outward current (data not shown), arguing against the involvement of a $\mathrm{Cl}$ conductance. Thus, the measured outward current was most likely contributed by a K conductance.

Because we showed previously that NO increases Ca levels in B5 neurons (Welshhans and Rehder, 2005) and others have demonstrated that $\mathrm{Ca}$-activated $\mathrm{K}$ currents are sensitive to NO (Ahern et al., 2002), we next determined whether a portion of the outward current was $\mathrm{Ca}$ dependent. In the presence of the Ca channel blocker $\mathrm{CdCl}_{2}(100 \mu \mathrm{M})$, the steady-state outward current at $+40 \mathrm{mV}$ was attenuated to $70 \pm 8 \%$ of control levels $(p \leq 0.001$, $n=3$ ) and precluded NOC-7 from additional inhibition of this current ( $p \geq 0.01, n=3$ ) (Fig. 5C). The fact that NOC-7 attenuated the steady-state $\mathrm{K}$ current to the same degree as inhibition of voltage-activated $\mathrm{Ca}$ currents with $\mathrm{CdCl}_{2}$ and the finding that the combined treatment with NOC-7 and $\mathrm{CdCl}_{2}$ had no additional effect suggested that both treatments acted through the same mechanism. Together, these results supported the hypothesis that NO exerted its effects by inhibiting a voltage-dependent Ca-sensitive K conductance.

\section{Evaluation of Ca-activated K currents}

There are two major types of Ca-sensitive K currents: BK-like channels are $\mathrm{Ca}$ activated and voltage sensitive, whereas SK-like channels are $\mathrm{Ca}$ activated but voltage insensitive (Sah and Faber, 2002). To determine whether the effects of NO on neuronal ac-
A

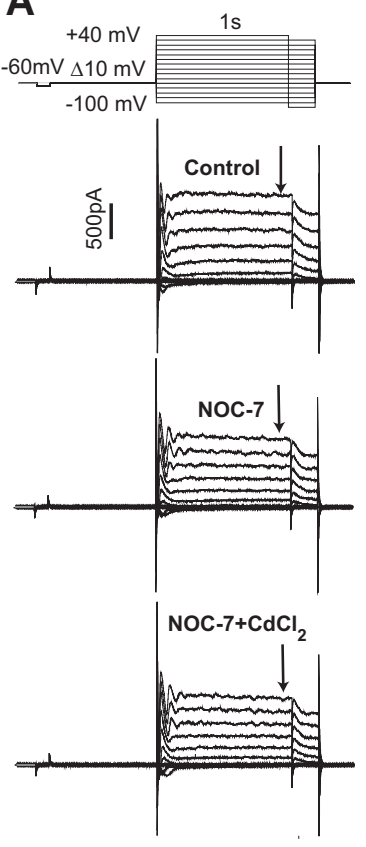

B
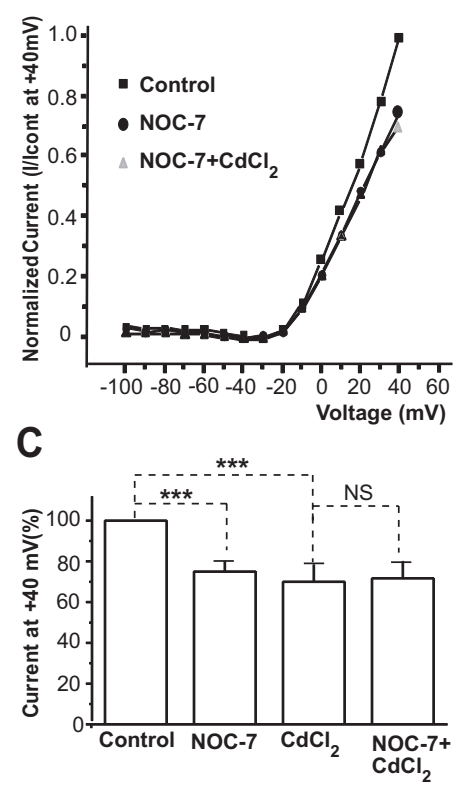

Figure 5. NOC-7 attenuates Ca-activated K currents. $\boldsymbol{A}$, Representative recording of total outward currents immediately before (control), 15 min after treatment with NOC-7 (100 $\mu \mathrm{M})$, and 1 min after treatment with $\mathrm{NOC}-7(100 \mu \mathrm{M})$ plus $\mathrm{CdCl}_{2}(100 \mu \mathrm{M})$. Currents were measured in whole-cell voltage-clamp mode from a holding potential of $-60 \mathrm{mV}$. Voltage was stepped from -100 to $+40 \mathrm{mV}$ in $10 \mathrm{mV}$ increments, and currents were measured $980 \mathrm{~ms}$ into the voltage step (arrow). $\boldsymbol{B}, I-V$ plot for control, NOC-7-treated and NOC-7 plus $\mathrm{CdCl}_{2}$-treated neurons measured as in $\boldsymbol{A}$ and normalized to the amount of control current at $+40 \mathrm{mV}$. Notice the high resistance at voltages between -100 and $-20 \mathrm{mV}$ and an increasing outward current between -20 and $+40 \mathrm{mV}$. C, NOC-7 significantly inhibited the steady-state outward current (measured at voltage steps to $+40 \mathrm{mV})$. The $\mathrm{Ca}$ channel blocker $\mathrm{CdCl}_{2}(100 \mu \mathrm{m})$ reduced the outward current to the same degree as $\mathrm{NOC}-7$, and the addition of $\mathrm{CdCl}_{2}$ in the presence of NOC-7 had no additional effect.

tivity and excitability could be explained by inhibition of such Ca-sensitive $\mathrm{K}$ conductances, we used specific inhibitors of BK and SK channels, IBTX and apamin (Habermann, 1984), respectively. Both inhibitors were used previously to inhibit their respective Ca-activated channels in snails (Schrofner et al., 2004; Vatanparast and Janahmadi, 2009) and we evaluated their effects on (1) the steady-state K outward conductance, (2) spontaneous spiking activity, (3) amplitude and duration of AHP, and (4) their ability to mimic and/or prevent the NOC-7-induced transient increase in firing activity and subsequent silencing.

\section{IBTX-sensitive K conductance or BK-like channels mediate} NO-induced inhibition of the outward current

Bath application of IBTX (300 nM) resulted in the partial inhibition of the steady-state $\mathrm{K}$ current, suggesting that part of the outward current was through a BK-like channel (Fig. 6A). The subsequent addition of NOC-7 to IBTX-treated neurons had no additional effect on the current (Fig. 6A). IBTX (300 nM) significantly inhibited the outward current to $63 \pm 8 \%$ of the control level ( $n=4, p \leq 0.001)$, whereas IBTX plus NOC-7 resulted in an inhibition to $60 \pm 9 \%(n=4, p \leq 0.001)$ (Fig. $6 B)$. There was no significant difference between treatment with IBTX and IBTX plus NOC-7, indicating that NOC-7 might have inhibited the steady-state outward current through an IBTX-sensitive BK channel.

Given the presence of an IBTX-sensitive BK-like conductance in B5 neurons, we next evaluated the ability of IBTX to affect the 
A

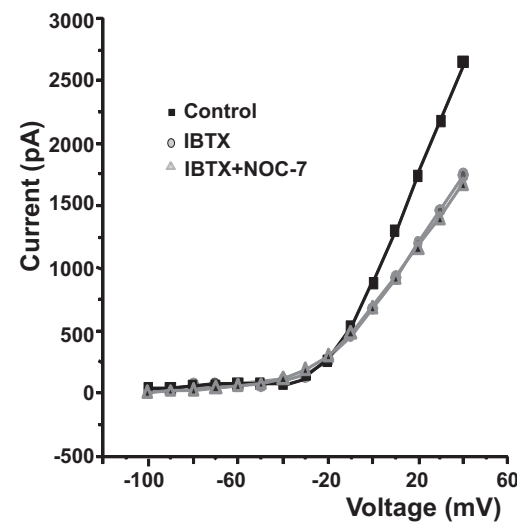

B

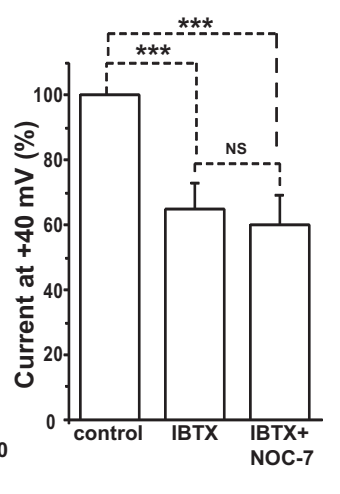

Figure 6. NOC-7 attenuates a steady-state outward current by inhibiting an IBTX-sensitive conductance. $A$, Total outward current under control condition, 30 min after IBTX ( $300 \mathrm{nM})$, and after IBTX (300 nM) plus NOC-7 (100 $\mu \mathrm{m})$. I-V curves were measured as in Figure 5 (holding potential of $-60 \mathrm{mV}$, voltage steps of $1 \mathrm{~s}$ duration from -100 to $+40 \mathrm{mV}$ in $10 \mathrm{mV}$ increments). $\boldsymbol{B}$, Quantification of data such as in $\boldsymbol{A}$. Iberiotoxin significantly attenuated the outward current measured at $+40 \mathrm{mV}(n=4, p \leq 0.001)$, and subsequent addition of NOC-7 (100 $\mu \mathrm{M})$ had no additional inhibitory effect on the outward current measured at $+40 \mathrm{mV}$.

spontaneous firing activity of B5 neurons. IBTX (300 nM) treatment resulted in an immediate increase in the firing rate of B5 neurons $(n=4)$, and the firing rate remained elevated for at least $30 \mathrm{~min}$ before returning to pretreatment levels (Fig. 7A). IBTX also resulted in significant spike broadening, increasing the halfwidth of spontaneous APs by $35 \pm 3 \%(p \leq 0.001, n=4)$ from $18.8 \pm 0.2 \mathrm{~ms}$ before to $25.4 \pm 0.3 \mathrm{~ms} 20 \mathrm{~min}$ into the treatment (Fig. 7A).

Blocking IBTX-sensitive conductance also had a strong effect on evoked AHPs. AHPs evoked by depolarizing current injections of $1 \mathrm{nA}$ for $1 \mathrm{~s}$ were decreased in amplitude by $30 \pm 8 \%(n=$ $4, p \leq 0.005)$ after treatment with IBTX when compared with controls (Fig. $7 B, C$ ). Interestingly, subsequent treatment with NOC-7 $(100 \mu \mathrm{M})$ for 20 min further decreased the AHP amplitude to $18 \pm 5 \%$ of control levels ( $p \leq 0.001, n=4)$, suggesting that NO affected the AHP by yet another mechanism.

Given that NOC-7 was able to further reduce the AHP in the presence of IBTX, we next investigated whether NOC-7 would be able to affect the spiking rate of IBTX-treated neurons as well. Once the firing rate had returned to pre-IBTX treatment levels, NOC-7 caused a transient increase in the firing rate and subsequently resulted in silencing of the firing activity $(n=5)$ (Fig. $7 D$ ), reminiscent of its effects on untreated neurons. Thus, although we have demonstrated that the inhibition of BK-like channels resulted in a transient increase in the firing rate of B5 neuron, much as after treatment with NOC-7, NOC-7 obviously acted through another pathway to silence neurons and, in addition to BK-like channels, could also have acted through another mechanism to increase spiking activity.

\section{Apamin-sensitive K conductance or SK-like channels mediate} NO-induced increase in spiking and subsequent silencing Given that NOC-7 affected neuronal firing and AHP amplitude in the presence of IBTX, we next investigated whether NOC-7, in addition to acting on BK-like channels, might also act on $\mathrm{Ca}$ dependent, voltage-insensitive SK-like channels. Treatment with the specific SK channel blocker apamin $(0.6 \mu \mathrm{M})$ resulted in transient depolarization and increase in spiking activity that was followed by long-term silencing after $\sim 10 \mathrm{~min}$ (Fig. $8 A$ ). Unlike treatment with NOC-7 and IBTX, apamin did not induce spike broadening (data not shown). Thus, treatment with apamin mimicked the effect of NOC-7 on depolarization, increased firing rate, and subsequent silencing.

In the majority of mammalian neurons, the main function of SK channels is ascribed to the generation of AHP (Sah and Faber, 2002; Pedarzani and Stocker, 2008) and SK channel-mediated currents are generally evaluated as the part of the AHP that is sensitive to apamin (Bond et al., 2005). Thus, we used whole-cell current-clamp recordings to generate AHPs and to measure the effect of apamin on K channels. The treatment with apamin reduced the depolarization-evoked AHP after current injection (1 $\mathrm{nA}, 1 \mathrm{~s}$ ) (Fig. $8 \mathrm{~B}$ ), suggesting that the apamin-sensitive conductance strongly contributed to the AHP in B5 neurons. The amplitude of AHPs (measured $300 \mathrm{~ms}$ after the end of the depolarizing pulse) was reduced from $-7.1 \pm 1.0 \mathrm{mV}$ before treatment (control) to $-2.5 \pm 0.5 \mathrm{mV}$ after apamin-induced silencing, a reduction of $65 \%(p \leq 0.001, n=4)$ (Fig. $8 C)$. The treatment with NOC-7 after apamin-induced silencing had an additional significant inhibitory effect on the amplitude of AHP, which was reduced to $-1.3 \pm 0.3 \mathrm{mV}(p \leq 0.005, n=4)$.

Because apamin appeared to have the same effect on spiking as treatment with NOC-7, we next investigated whether NOC-7 would have an effect on apamin-silenced neurons. Interestingly, once silenced, apamin-treated neurons showed no response to NOC-7 $(n=4)$ (Fig. $8 D$ ), suggesting that the apamin-sensitive conductance was the major target of $\mathrm{NO}$ in mediating the effect of NOC-7 on the intrinsic neuronal activity of B5 neurons.

In summary, our results suggest that the effects of NOC-7 on the intrinsic firing activity of B5 neurons are primarily mediated via inhibition of SK-like Ca-sensitive $\mathrm{K}$ channels and partially via inhibition of BK channels, contributing to the initial increase in firing rate and spike broadening.

\section{Effects of cellular NO release on electrical properties}

Although NO donors, such as NOC-7, have played a significant role in the investigation of NO signaling, it has become increasingly clear that biological effects of NO have to be interpreted in light of the actual physiological release of NO from cells and tissues in vivo. We previously developed a sender-receiver paradigm, in which we investigated the effect of physiological NO release from a single neuron on growth cone motility of other neurons. This study showed that the NO release from a single neuron can be sufficient to have a physiological effect on another neuron located up to $100 \mu \mathrm{m}$ away (Tornieri and Rehder, 2007). Here we used the same senderreceiver paradigm to investigate whether NO release from a single cell (sender) has physiological effects on the electrical properties of neighboring neurons (receivers). Identified B5 neurons, known to express the NO-synthesizing enzyme NOS, were used as sources of constitutive NO production and positioned $\sim 15-20$ $\mu \mathrm{m}$ from the soma of a receiver that was recorded in whole-cell current-clamp mode (Fig. 9A). Senders resulted in silencing of receivers in seven of seven experiments within $12-20 \mathrm{~min}$, just as observed above with NOC-7 but with a slower time course. This might be attributed to the smaller concentration of $\mathrm{NO}$ released from the sender compared with NOC-7. NOC-7 caused a biphasic response with a transient increase in firing rate followed by silencing, Similarly, senders caused an initial transient increase of spiking in five of seven receivers, and the increase seen ranged from pronounced (Fig. 9B, top) to weak (Fig. 9B, bottom). In the remaining two of seven experiments with $B 5$ senders, receivers were silenced within 12-15 min without an initial transient in- 
crease in spiking activity. Subsequent removal of B5 senders from receivers did not result in the recovery of tonic spiking within $1 \mathrm{~h}$, suggesting that the changes in electrical activity induced by the sender were long lasting (data not shown).

Because we had shown previously that NOC-7 resulted in the reduction of depolarization-evoked AHPs, we hypothesized that NO release from a physiological source might have the same effect. This was indeed the case, because AHPs elicited with the depolarizing current injection protocol (1 nA, 1s) after sender-induced silencing were significantly reduced to $57 \pm 13 \%(p \leq 0.005, n=4)$ (Fig. 9C). To verify that it was indeed NO and not another substance that was responsible for the effects of sender neurons on electrical activity, we used two controls: (1) B19 neurons, which show no or only very faint immunoreactivity to nNOS (Van Wagenen and Rehder, 2001) and were shown not to release sufficient amounts of NO to affect other neurons (Tornieri and Rehder, 2007) and (2) B5 neurons in which nNOS was knocked down by an RNAi approach. As demonstrated previously in the fresh-water snail Lymnaea (Korneev et al., 2002), bath application of several hundred base pairs of doublestranded RNA in vitro resulted in the degradation of targeted RNAs. We used this approach with double-stranded RNA produced from $H$. trivolvis as detailed below.

Treatment of B5 neurons with HeliNOS RNAi abolishes the effect of an NO sender on its target

Before determining the effectiveness of the NOS RNAi approach in electrophysiological experiments, we first investigated its effectiveness in reducing the amount of NOS expressed in senders. Incubation of senders for $24 \mathrm{~h}$ with HeliNOS RNAi (1 $\mu \mathrm{g} / \mathrm{ml}$ ) significantly decreased the expression of nNOS by $80 \pm 5 \%$ compared with control neurons incubated only with vehicle ( $p \leq 0.001, n=19$ and $n=14$, respectively) (Fig. $10 B, D$ ). To independently verify the effectiveness of the NOS knockdown, we used the sender-receiver paradigm (Tornieri and Rehder, 2007) to test the effect of physiological NO release on filopodial motility. Growth cone filopodia are exquisitely sensitive to NO and a valuable assay system for effects of NO. Stimulation with NO resulted in a transient elongation of filopodia, whereas senders incubated with HeliNOS RNAi $(1 \mu \mathrm{g} / \mathrm{ml})$ for $24 \mathrm{~h}$ had no effect on filopodia length (Fig. $10 \mathrm{E}$ ), suggesting that the knockdown was sufficient to block physiological effects of NO on growth cone filopodia.

To confirm that the effect of NO senders on electrical properties of receivers was mediated through NO, we preincubated
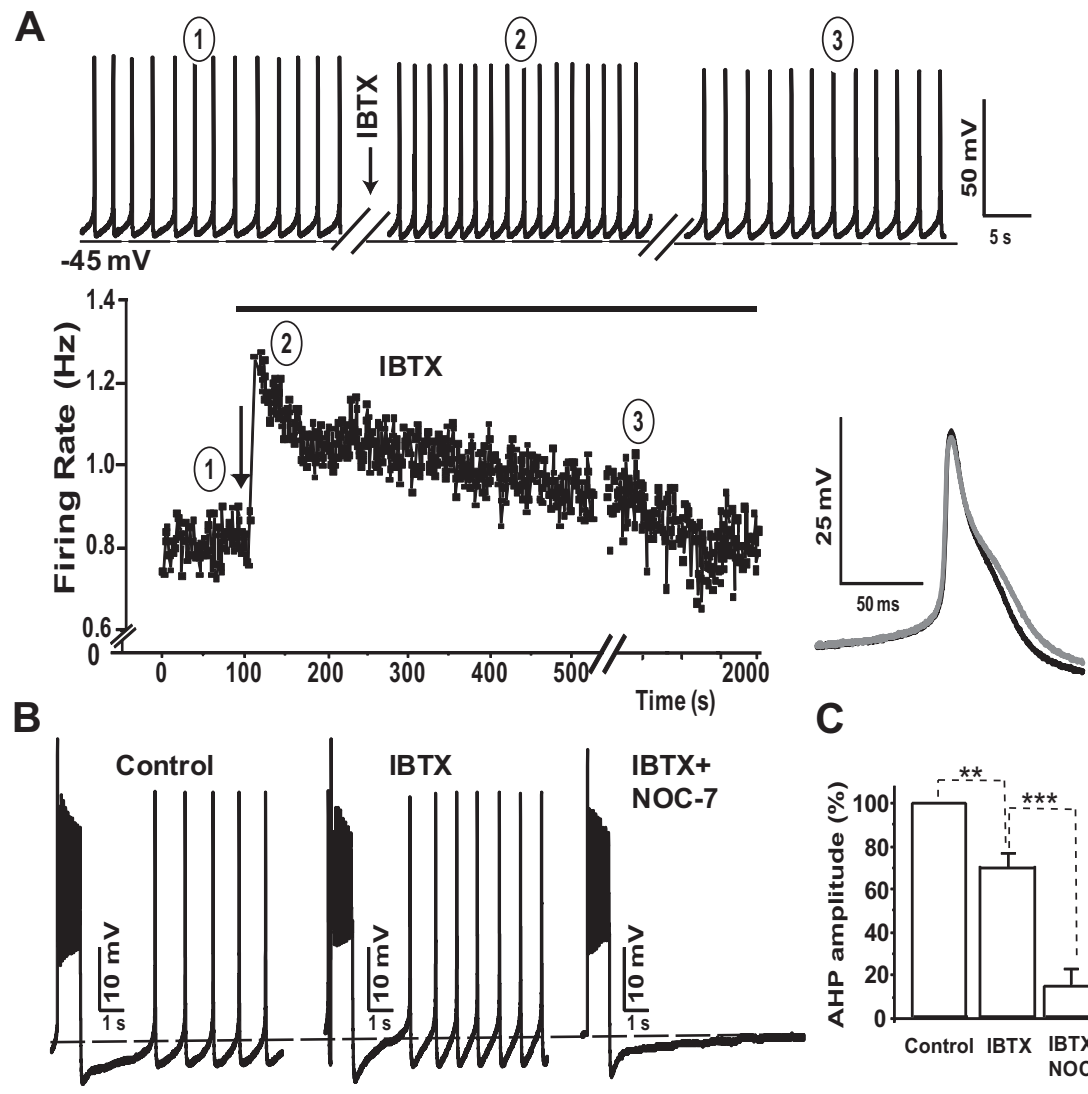

C
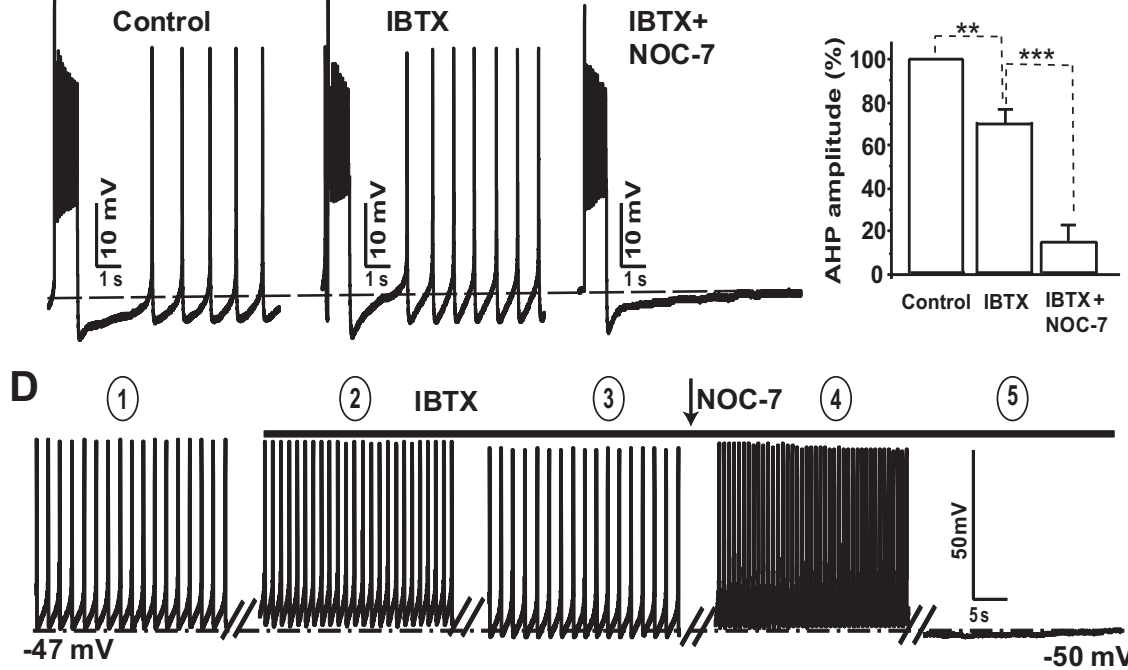

Figure 7. Inhibition of IBTX-sensitive $K$ channels transiently activates firing activity and decreases the AHP of B5 neurons. $A$, Top illustrates a representative trace of a B5 neuron firing spontaneously (1), during the first minute of treatment with IBTX (2), and 30 min after treatment (3). Bottom shows the continuous firing rate of the same neuron, highlighting the transient IBTX-induced peak in firing rate and the slow recovery to pretreatment values during the next $30 \mathrm{~min}$. The right illustrates the broadening of spontaneous APs from control levels of $18.79 \pm 0.03 \mathrm{~ms}(n=40$; black line) to $25.42 \pm 0.06 \mathrm{~ms}(n=40) 20$ min after treatment with IBTX (gray line), an increase in AP half-width of $35 \%$ ( $p \leq 0.001, n=40)$. B, Effect of IBTX and IBTX plus NOC-7 on AHP amplitude after current injection-evoked depolarization ( $+1 \mathrm{nA}, 1 \mathrm{~s})$. $\boldsymbol{C}$, Quantification of experiments shown in $\boldsymbol{B}$. IBTX caused a significant decrease in the amplitude of AHPs $(n=4, p \leq 0.005)$, and subsequent treatment with NOC -7 (100 $\mu \mathrm{M})$ resulted in an additional significant inhibition of AHP amplitude $(n=4, p \leq 0.001)$. AHP amplitude was measured $200 \mathrm{~ms}$ after the end of the depolarizing pulse. $D$, Inhibition of IBTX-sensitive $K$ channels did not block the effects of NOC-7 on firing activity of B5 neurons. Recording shows spiking activity 20 s before treatment with IBTX (1), during the first minute with IBTX (2), and 30 min with IBTX (3). The additional treatment with NOC-7 (100 $\mu \mathrm{m}$; arrow) 30 min into the treatment with IBTX transiently increased firing activity (4) and silenced activity a few minutes later (5). Gray line shows the continued presence of IBTX in the bath.

senders for $24 \mathrm{~h}$ with HeliNOS RNAi before testing. Senders incubated with HeliNOS RNAi had no effect on the firing rate of receiver neurons (Fig. $11 A$, top), independently supporting the notion of an effective knockdown of NOS. The other control, B19 neurons, also had no effect on the spontaneous firing rate of B5 neurons (Fig. 11 A, bottom). Together, these results suggested that the silencing effect observed with $\mathrm{B} 5$ senders was attributable to NO.

The analysis of AHPs in response to a depolarizing pulse (1 $\mathrm{nA}, 1 \mathrm{~s}$ ) provided independent support that the RNAi approach functionally knocked down nNOS in B5 senders. The AHP am- 
A
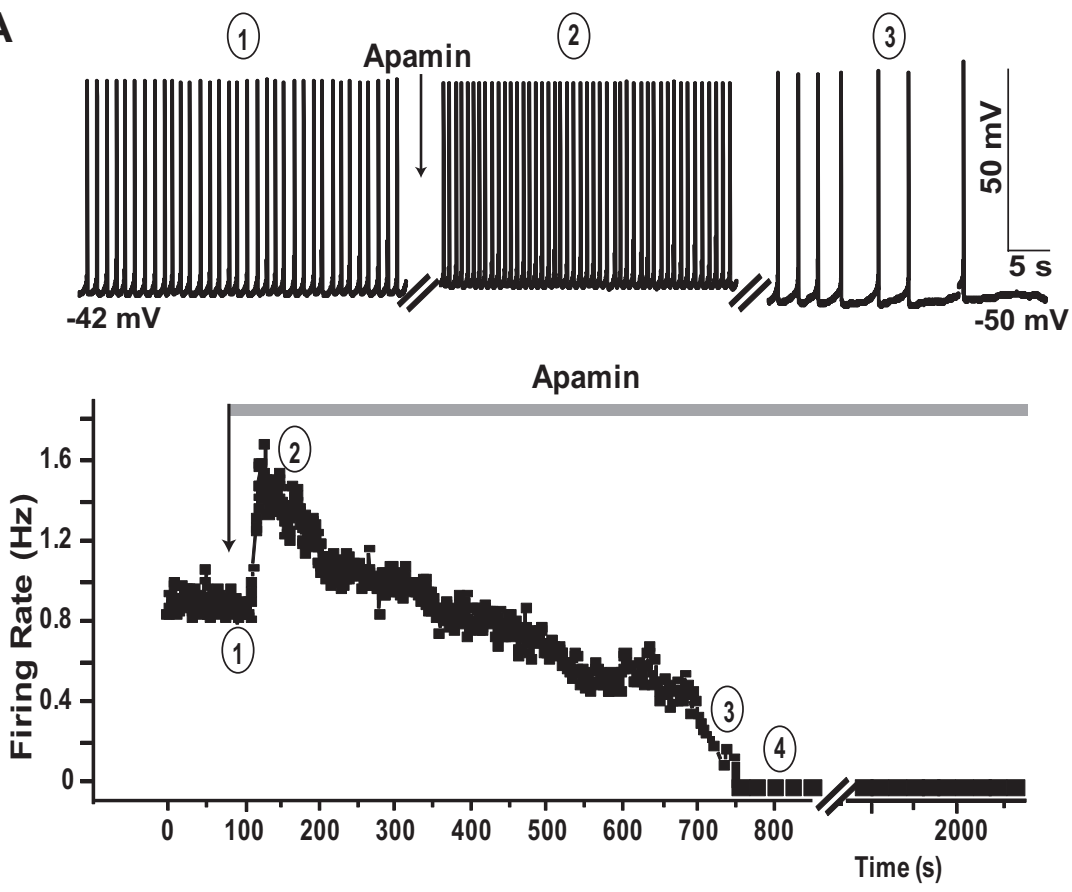

B
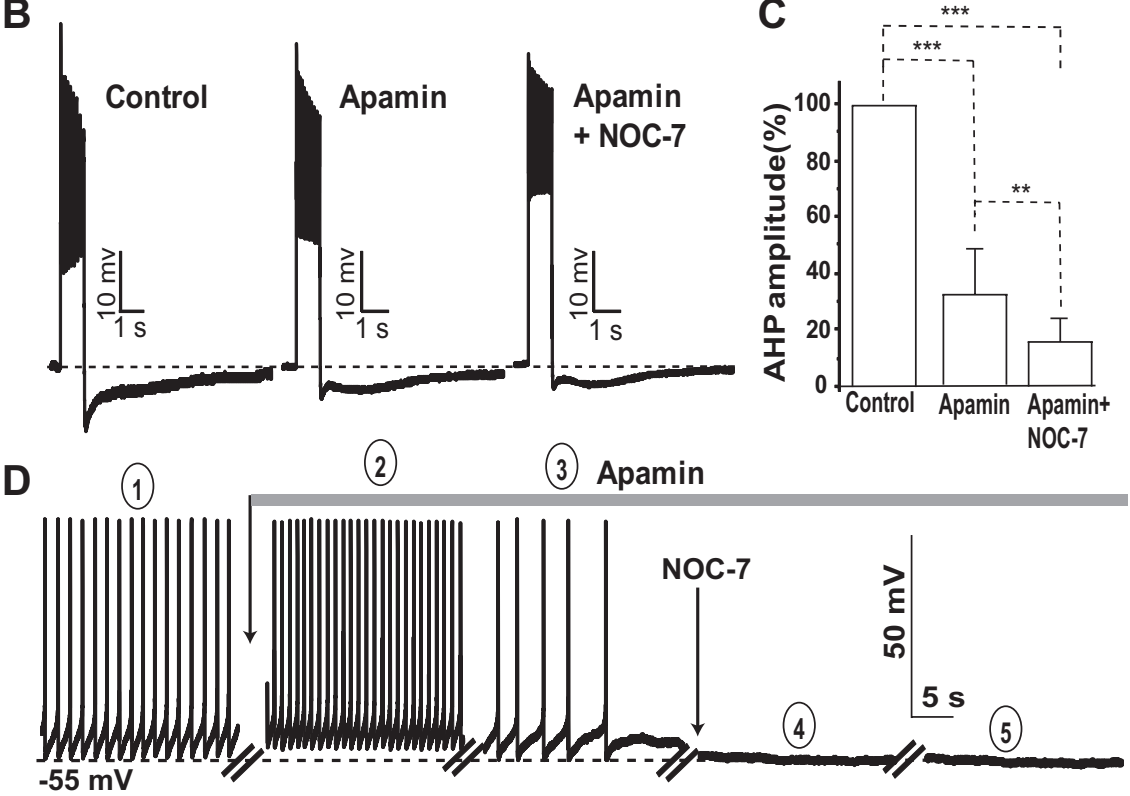

Figure 8. Inhibition of apamin-sensitive $K$ channels mimics the $N 0$-induced effects on neuronal firing. $A$, Top, A representative recording of a neuron showing tonic spontaneous firing activity of $\sim 0.9 \mathrm{~Hz}(1)$, a transient depolarization and increase in spiking to $\sim 1.5 \mathrm{~Hz}$ after treatment with apamin (2), and cessation of spontaneous firing at hyperpolarized membrane potential $(\Delta V \sim 8$ $\mathrm{mV}$ ) after $10 \mathrm{~min}$ in apamin (3). Bottom, Plot of firing rate over time for neuron shown above. $\boldsymbol{B}$, Apamin inhibited the AHP that was evoked after APs induced by positive current injection (1 nA, $1 \mathrm{~s})$. Addition of NOC-7 to apamin-treated neurons caused an additional decrease in AHP amplitude. $\boldsymbol{C}$, Quantification of experiments $(n=4)$ such as the one shown in $\boldsymbol{B}$. AHP was measured 300 $\mathrm{ms}$ after the end of the depolarizing step. $\boldsymbol{D}$, Inhibition of apamin-sensitive $K$ channels blocks the effects of NOC -7 on firing activity. Representative recording $(n=6)$ showing spontaneous spiking activity $20 \mathrm{~s}$ before apamin addition (1), depolarization and increased firing after $1 \mathrm{~min}$ (2), and decrease in firing rate and silencing after $30 \mathrm{~min}$ in apamin (3). Treatment with NOC-7, once the neuron has been silenced by apamin, had no additional immediate effect on electrical activity (4), and the neuron remained silent for at least 30 min after addition of NOC-7 (5). Gray line indicates the continued presence of apamin in the bath. Arrow depicts the application of NOC-7.

plitudes in untreated control neurons, in receivers treated with B19 senders, and in receivers of nNOS RNAi-treated B5 senders were not significantly different (Fig. $11 \mathrm{~B}$ ), indicating that $\mathrm{NO}$ is responsible for the modulatory effect of $\mathrm{B} 5$ senders on electrical activity of B5 neurons. Together, these data demonstrated that
NO released from a cellular source can affect the electrical properties of $\mathrm{NO}$ sensitive neurons growing in its surrounding and that the RNAi approach used here can be used effectively in cell culture applications.

\section{Discussion}

NO modulates electrical properties in different cell types, both in vivo and in vitro (Ferraro and Sardo, 2004; Garthwaite, 2008). Here we describe a novel mechanism by which NO significantly alters the excitability of an identified gastropod neuron, namely by inhibiting BK- and SK-like K conductances. Moreover, by using a single neuron as an NO source, we demonstrate that physiological release of NO is sufficient to significantly affect electrophysiological responses in synaptically unconnected neurons. Last, we show that the electrophysiological responses seen using a cellular NO source are very similar to responses observed after application of chemical NO donors.

\section{SK-type and BK-type potassium} channels mediate long- and short-term effects of NOC-7

Treatment with the NO donor NOC-7 resulted in membrane depolarization, a transient increase in firing frequency, spike broadening, and subsequent silencing of spiking activity that coincided with the inhibition of Ca-activated K currents. Surprisingly, all these effects, except spike broadening, were seen after inhibition of SK-like K conductances with apamin, suggesting that $\mathrm{NO}$ affected firing activity by inhibiting an SK-like conductance. Interestingly, the initial increase in spiking activity and spike broadening, as well as a decrease in the steady-state $\mathrm{K}$ current, were also mimicked by IBTX, indicating a BK-like channel as a target of $\mathrm{NO}$ as well. $\mathrm{BK}$ and SK channels gate two major types of Ca-sensitive K currents. Whereas BKlike channels are $\mathrm{Ca}$ activated and voltage sensitive, SK-like channels are Ca activated and voltage insensitive (Sah and Faber, 2002). Both types of channels have been localized in gastropod neurons with antibodies developed for mammalian BK and SK channels (Azanza et al., 2008), suggesting a fair degree of conservation of these channels across phyla.

We demonstrated previously that NOC-7 treatment resulted in a transient increase in intracellular $\mathrm{Ca}$ through the release of $\mathrm{Ca}$ from intracellular stores and via voltage-gated $\mathrm{Ca}$ channels (Van Wagenen and Rehder, 1999; Welshhans and Rehder, 2007). The inhibition of Ca-activated K channels by NOC-7 resulted in depolarization and the subsequent opening of 


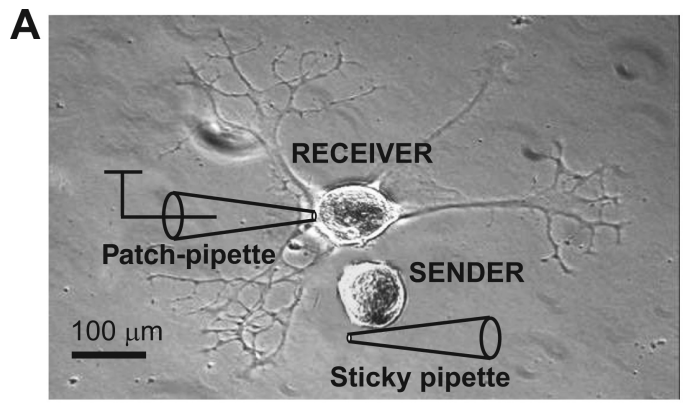

B

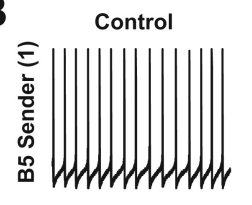

20 seconds after 20 minutes after
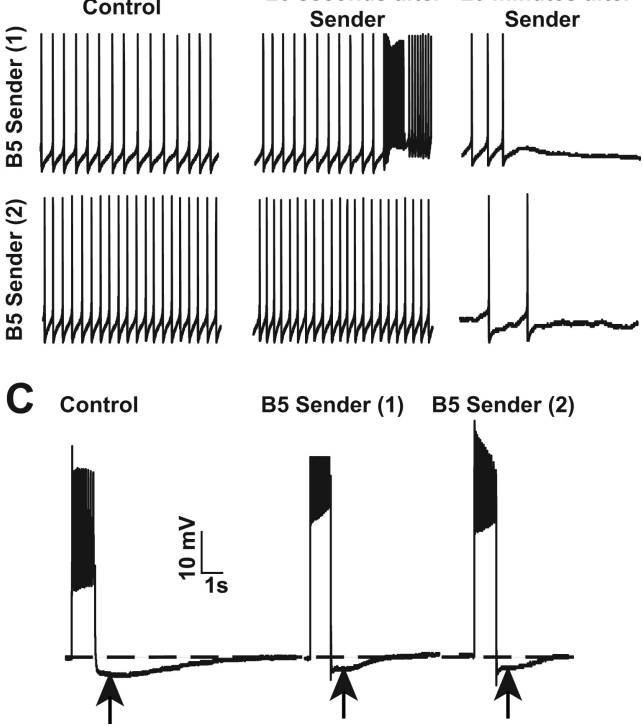

Figure 9. NO senders mimic the effects of NOC-7 on electrical activity and AHP of receiver neurons. $\boldsymbol{A}$, Experimental setup of a sender-receiver experiment. A sender neuron, attached to a poly-L-lysine-coated microelectrode, was moved into the vicinity of the soma of a receiver neuron. The response of the receiver was recorded with a patch-clamp electrode. $\boldsymbol{B}$, Representative recordings from two receivers demonstrating the changes in firing activity after sender $B 5$ was moved close to the soma of receiver. Recordings show spontaneous activity before (control), $20 \mathrm{~s}$, and $20 \mathrm{~min}$ after the sender was moved close to the receiver. Recording on top shows an example in which the receiver showed a strong transient increase in spiking and subsequent silencing, whereas the neuron on the bottom only showed a very small increase in the firing rate before it was silenced. C, Example of AHPs recorded from a control receiver (no exposure to sender; left) and after silencing by a sender (middle, right; numbers correspond to neurons in $\boldsymbol{B}$ ).

voltage-gated Ca channels. The resulting increase in cytosolic $\mathrm{Ca}$ might account for part of the $\mathrm{Ca}$ influx seen in our previous studies. The increase in firing rate in response to NOC-7 would likely cause an additional increase in intracellular $\mathrm{Ca}$ given that Ca ions are a major contributor to the APs in B5 neurons and that NOC-7 also resulted in spike broadening.

Most likely, NOC-7 and IBTX broadened APs directly via inhibition of voltage-dependent Ca-activated K currents. Any indirect broadening of spikes via modification of other channels by depolarization would have been revealed by other treatments resulting in comparable depolarization. Apamin, for example, although having a depolarizing effect, did not result in spike broadening, suggesting that the effect of NOC-7 on spike broadening likely occurred through the inhibition of a voltagedependent Ca-activated $\mathrm{K}$ conductance, such as an IBTXsensitive, BK-like channel.

Our finding that apamin induced an increase in the firing rate without broadening of APs was consistent with the effects of apamin on APs described previously (Gao and ZiskindConhaim, 1998). Apamin-induced silencing was consistent also
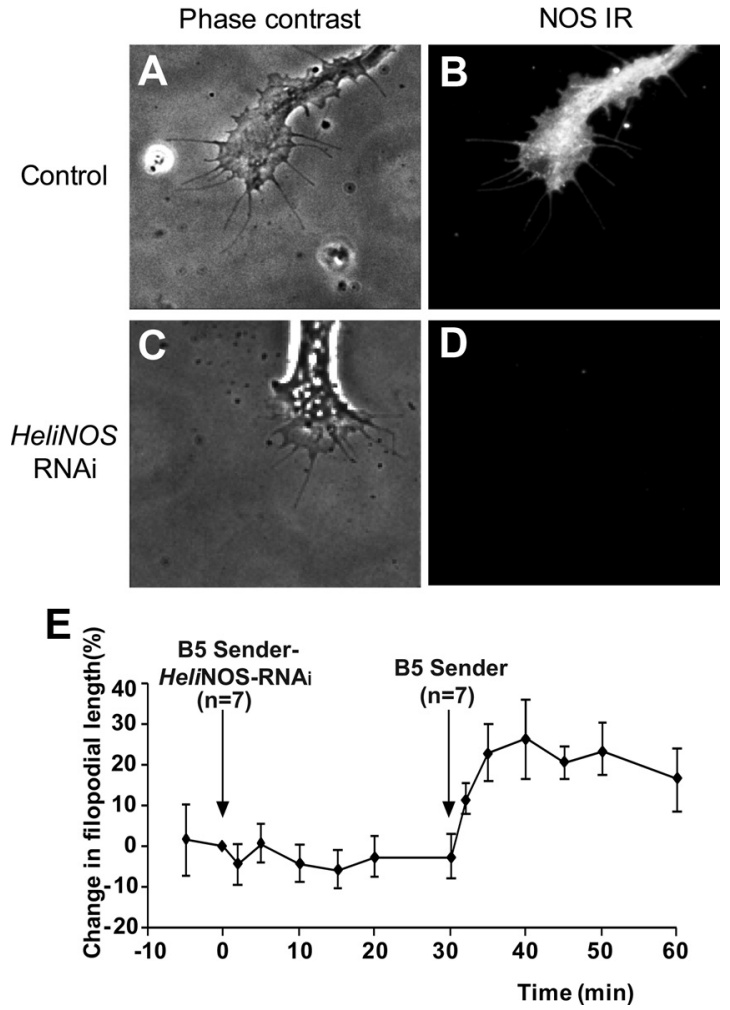

Figure 10. RNAi treatment knocks down NOS expression and fully blocks the effect of NO on growth cone morphology. $\boldsymbol{A}-\boldsymbol{D}$, Immunocytochemistry with an antibody against brain NOS strongly stained growth cones and neurites of control senders grown in vitro $(\boldsymbol{B})$ but very little to no staining was observed in HeliNOS RNAi-treated neurons $(\boldsymbol{D})$. The same growth cones were shown in phase-contrast images in $\boldsymbol{A}$ and $\boldsymbol{C}$. IR, Immunoreactivity. $\boldsymbol{E}$, RNAi-treated senders moved close to receiver growth cones $(\sim 30-60 \mu \mathrm{m})$ growing in cell culture had no effect on filopodial morphology, whereas control senders elicited a rapid and long-lasting elongation of filopodia.

with the critical role of SK channels in the maintenance of tonic spiking activity (Bond et al., 2005). Moreover, apamin treatment prevented NO from having additional effects on electrical activity, indicating that the effects of NO on neuronal excitability could be explained by its effect on apamin-sensitive K channels. Considering the above, we concluded that NOC-7 inhibited Caactivated $\mathrm{K}$ channels by a yet unknown mechanism that also prevented or decreased the normal activation of BK- and SK-like $\mathrm{K}$ channels by $\mathrm{Ca}$.

AHPs are critical for setting the spontaneous spiking activity and are important in determining neuronal excitability in general (Hille, 2001; Disterhoft and Oh, 2006). Ca-activated K channels are known to help repolarize the membrane potential and are primarily responsible for the AHP (Hille, 2001). BK- and SK-like channels contribute to the AHP in different ways (Köhler et al., 1996; Sah and Faber, 2002). BK channels contribute to the initial phase of the AHP (Shao et al., 1999), and SK channels contribute to the medium AHP that typically lasts for several hundred milliseconds (Sah and Faber, 2002; Vatanparast and Janahmadi, 2009). We demonstrated that NO decreased the AHPs after rebound APs and strong depolarizing current pulses. Consequences of AHP inhibition are a faster recovery of the membrane potential, which would allow neurons to fire at a higher frequency and result in increased intrinsic excitability. The specific inhibitors of SK-like channels, apamin, and BK-like channels, IBTX, also significantly reduced the AHP and transiently increased firing frequency. Interestingly, the AHP of apamin- 
A
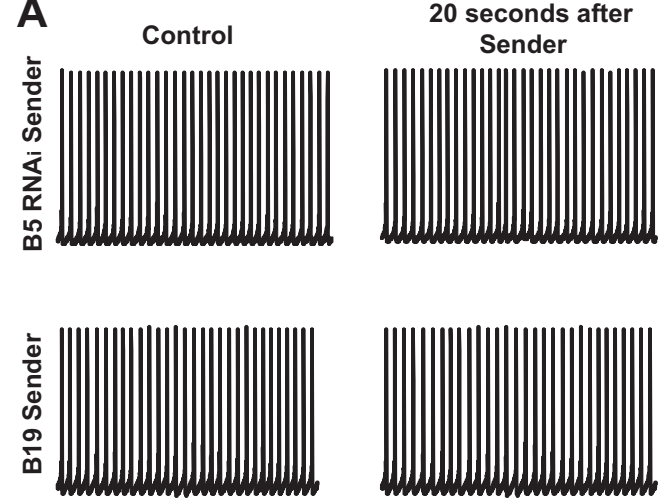

B
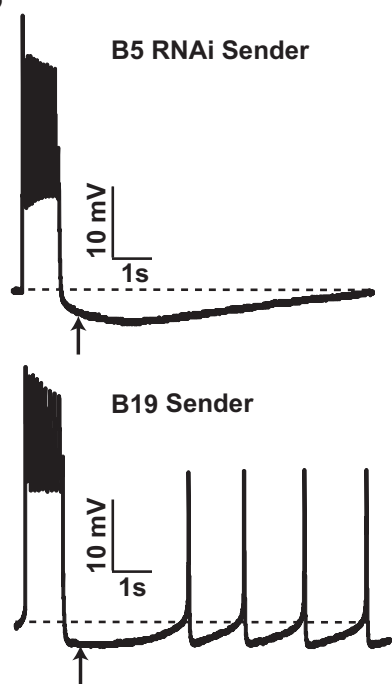

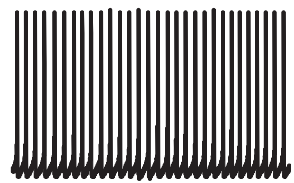

20 minutes after Sender
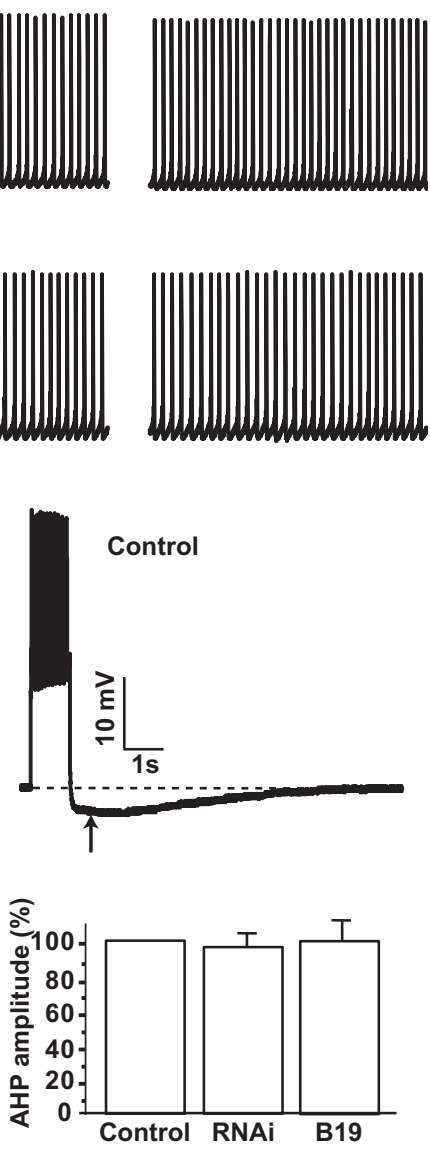

Figure 11. NOS RNAi-treated senders and B19 senders do not affect the electrical activity of $B 5$ receivers. $A$, Traces of recordings from receivers before (control), $20 \mathrm{~s}$ after, and 20 min after moving an RNAi-treated sender (top) or B19 sender (bottom) in close proximity $(\sim 20 \mu \mathrm{m})$ to the soma of the recorded neuron. Note that these treatments had no effect on the electrical activity of the receiver. $\boldsymbol{B}$, Large AHPs were elicited in untreated receivers, and the amplitude of the AHPs was not affected after pairing the receiver with RNAi-treated B5 senders or B19 senders for $20-30 \mathrm{~min}$. AHPs were measured $300 \mathrm{~ms}$ after the end of the depolarization step.

treated neurons could be reduced further by NOC-7, an effect that was likely attributable to the IBTX-sensitive component of the AHP, both of which contribute to the compound AHP.

The early effect of NOC-7 was a transient increase in firing rate and the later effect was silencing. This silencing was long lasting and reminiscent of the long-term effects of NO on the modulation of synaptic strength in models of synaptic plasticity (Zhuo and Hawkins, 1995; Feil and Kleppisch, 2008). Neuronal silencing by NO could represent a novel NO-dependent form of homeostatic plasticity caused by long-term changes in the functional expression of outward $\mathrm{K}$ currents, particularly $\mathrm{Ca}-$ activated $\mathrm{K}$ channels that, in the intact nervous system, would result in altered responses to incoming synaptic and nonsynaptic inputs and gate synaptic outputs.

\section{Possible mechanisms of SK channel regulation}

Although we presently do not know how NO inhibits Caactivated $\mathrm{K}$ channels in $\mathrm{B} 5$ neurons, several $\mathrm{Ca}$-dependent and Ca-independent mechanisms by which these channels can be modulated have been described. Ca-activated $\mathrm{K}$ channels can be inhibited by prolonged increases in intracellular $\mathrm{Ca}$. For instance, prolonged stimulation of group 1 metabotropic glutamate receptors in hippocampal CA1 neurons triggers intracellular Ca re-

lease, which results in the inhibition of the $\mathrm{AHP}$ and its underlying Ca-activated $\mathrm{K}$ currents (Mannaioni et al., 2001; Young et al., 2008). We demonstrated previously that $\mathrm{NO}$ induces an increase in cytosolic Ca via ryanodine channels (Welshhans and Rehder, 2005), raising the possibility of $\mathrm{Ca}$ release-mediated inhibition of AHP. Additionally, in amygdala pyramidal neurons, PKA phosphorylation primes SK channels for removal from the plasma membrane, bearing a striking resemblance with the removal of AMPA channels in long-term depression (Ziff, 2007). Moreover, a decrease in Ca sensitivity of SK channels has been reported to occur via CK2 kinase phosphorylation of calmodulin (Allen et al., 2007). Interestingly, inhibition of SK channels has been linked to serine phosphorylation of these channels (Kramár et al., 2004), raising the possibility of NO-induced PKG phosphorylation of these channels in B5 neurons (Welshhans and Rehder, 2005). Regardless of the signaling pathways, a prolonged downregulation of SK channels might be the general mechanism by which NO exerts its long-lasting modulatory effect on both intrinsic and synaptic neuronal plasticity.

\section{NO as a volume messenger}

The function of NO as a "volume" transmitter has been proposed in systems in which the source and target of NO are anatomically segregated (Gally et al., 1990; Wood and Garthwaite, 1994; Philippides et al., 2005). Modeling approaches have shown that NO will diffuse from its source of production and affect other neurons at considerable distances (Wood and Garthwaite, 1994; Philippides et al., 2005). We demonstrated that NO has physiological effects at distances of up to $100 \mu \mathrm{m}$ (Tornieri and Rehder, 2007). In the sender-receiver paradigm used here, soma-to-soma reset of neuronal excitability by the sender suggested that NO might regulate and coordinate the electrical activities of neighboring neurons that express $\mathrm{NO}$ targets in vivo. Our finding that $\mathrm{NO}$ served as a modulator of neuronal activity is supported by studies in other systems (Ahern et al., 2002; D'Yakonova and D'Yakonova, 2008). In the related gastropod Lymnaea, NO enhanced the serotonergic synapse between the cerebral giant cell and B4 neuron (Straub et al., 2007), whereas NO converted an inhibitory effect carried by glutamate into an excitatory effect (D'Yakonova and D'Yakonova, 2008). Modulating neuronal firing and silencing of neurons in circuits that generate motor behaviors can have strong effects on behavioral output and can even result in a switch between motor programs (Selverston et al., 1998). Thus, the intrinsic neuronal activity of neurons participating in the same or different neuronal circuits could be affected by NO synchronously, resulting in modulated circuit outputs. One example, in which NO was shown to initiate or modulate a motor output is feeding behavior in mollusks, although the location and the mechanism by which NO affects the feeding program are 
not fully understood (Moroz et al., 1993; Elphick et al., 1995; Kobayashi et al., 2000a,b; Hatcher et al., 2006).

There has been a long-standing debate about the physiological relevance of using chemical NO donors (Garthwaite, 2008). Our finding that both electrical properties and effects on growth cone morphology are regulated in a similar manner by chemical and physiologically released NO supports the usefulness of commercially available NO donors in studies of cellular physiology, although NO release from cellular sources is likely to produce a more physiological stimulus. Although NOC-7 and stimulation with a NO-releasing cell resulted in silencing, not all neurons treated with the NO sender showed the initial increase in firing, suggesting that the amounts and temporal dynamics of NO release from the two sources are likely different. Future studies will have to continuously measure and compare the amounts of $\mathrm{NO}$ release from single cells and from NO donors to better understand the temporal and spatial aspects of NO release, as well as their effects on different cellular targets.

\section{References}

Adams DJ, Smith SJ, Thompson SH (1980) Ionic currents in molluscan soma. Annu Rev Neurosci 3:141-167.

Ahern GP, Klyachko VA, Jackson MB (2002) cGMP and S-nitrosylation: two routes for modulation of neuronal excitability by NO. Trends Neurosci 25:510-517.

Allen D, Fakler B, Maylie J, Adelman JP (2007) Organization and regulation of small conductance $\mathrm{Ca}^{2+}$-activated $\mathrm{K}^{+}$channel multiprotein complexes. J Neurosci 27:2369-2376.

Azanza MJ, Pérez-Castejón C, Pes N, Pérez-Bruzón RN, Aisa J, Junquera C, Maestú C, Lahoz M, Martínez-Ciriano C, Vera-Gil A, Del Moral A (2008) Characterization by immunocytochemistry of ionic channels in Helix aspersa suboesophageal brain ganglia neurons. Histol Histopathol 23:397-406.

Bicker G (2005) STOP and GO with NO: nitric oxide as a regulator of cell motility in simple brains. Bioessays 27:495-505.

Bond CT, Maylie J, Adelman JP (2005) SK channels in excitability, pacemaking and synaptic integration. Curr Opin Neurobiol 15:305-311.

Clasadonte J, Poulain P, Beauvillain JC, Prevot V (2008) Activation of neuronal nitric oxide release inhibits spontaneous firing in adult gonadotropin-releasing hormone neurons: a possible local synchronizing signal. Endocrinology 149:587-596.

Cohan CS, Kater SB (1986) Suppression of neurite elongation and growth cone motility by electrical activity. Science 232:1638-1640.

Cohan CS, Welnhofer EA, Zhao L, Matsumura F, Yamashiro S (2001) Role of the actin bundling protein fascin in growth cone morphogenesis: localization in filopodia and lamellipodia. Cell Motil Cytoskeleton 48:109120.

Contestabile A, Ciani E (2004) Role of nitric oxide in the regulation of neuronal proliferation, survival and differentiation. Neurochem Int 45:903-914.

Disterhoft JF, Oh MM (2006) Learning, aging and intrinsic neuronal plasticity. Trends Neurosci 29:587-599.

D'Yakonova TL, D'Yakonova VE (2008) Modification of the effects of glutamate by nitric oxide $(\mathrm{NO})$ in a pattern-generating network. Neurosci Behav Physiol 38:407-413.

Elphick MR, Kemenes G, Staras K, O'Shea M (1995) Behavioral role for nitric oxide in chemosensory activation of feeding in a mollusc. J Neurosci 15:7653-7664.

Feil R, Kleppisch T (2008) NO/cGMP-dependent modulation of synaptic transmission. Handb Exp Pharmacol 2008:529-560.

Ferraro G, Sardo P (2004) Nitric oxide and brain hyperexcitability. In Vivo 18:357-366.

Fields RD, Neale EA, Nelson PG (1990) Effects of patterned electrical activity on neurite outgrowth from mouse sensory neurons. J Neurosci 10:2950-2964.

Frick A, Johnston D (2005) Plasticity of dendritic excitability. J Neurobiol 64:100-115.

Gally JA, Montague PR, Reeke GN Jr, Edelman GM (1990) The NO hypothesis: possible effects of a short-lived, rapidly diffusible signal in the devel- opment and function of the nervous system. Proc Natl Acad Sci U S A 87:3547-3551.

Gao BX, Ziskind-Conhaim L (1998) Development of ionic currents underlying changes in action potential waveforms in rat spinal motoneurons. J Neurophysiol 80:3047-3061.

Garthwaite J (2008) Concepts of neural nitric oxide-mediated transmission. Eur J Neurosci 27:2783-2802.

Habermann E (1984) Apamin. Pharmacol Ther 25:255-270.

Hatcher NG, Sudlow LC, Moroz LL, Gillette R (2006) Nitric oxide potentiates cAMP-gated cation current in feeding neurons of Pleurobranchaea californica independent of cAMP and cGMP signaling pathways. J Neurophysiol 95:3219-3227.

Hille B (2001) Ionic channels of excitable membranes. Sunderland, MA: Sinauer Associates.

Ibarretxe G, Perrais D, Jaskolski F, Vimeney A, Mulle C (2007) Fast regulation of axonal growth cone motility by electrical activity. J Neurosci 27:7684-7695.

Kobayashi S, Ogawa H, Fujito Y, Ito E (2000a) Nitric oxide suppresses fictive feeding response in Lymnaea stagnalis. Neurosci Lett 285:209-212.

Kobayashi S, Sadamoto H, Ogawa H, Kitamura Y, Oka K, Tanishita K, Ito E (2000b) Nitric oxide generation around buccal ganglia accompanying feeding behavior in the pond snail, Lymnaea stagnalis. Neurosci Res 38:27-34.

Koh HY, Jacklet JW (2001) Nitric oxide induces cGMP immunoreactivity and modulates membrane conductance in identified central neurons of Aplysia. Eur J Neurosci 13:553-560.

Köhler M, Hirschberg B, Bond CT, Kinzie JM, Marrion NV, Maylie J, Adelman JP (1996) Small-conductance, calcium-activated potassium channels from mammalian brain. Science 273:1709-1714.

Korneev SA, Kemenes I, Straub V, Staras K, Korneeva EI, Kemenes G, Benjamin PR, O'Shea M (2002) Suppression of nitric oxide (NO)dependent behavior by double-stranded RNA-mediated silencing of a neuronal NO synthase gene. J Neurosci 22:RC227(1-5).

Kramár EA, Lin B, Lin CY, Arai AC, Gall CM, Lynch G (2004) A novel mechanism for the facilitation of theta-induced long-term potentiation by brain-derived neurotrophic factor. J Neurosci 24:5151-5161.

Mannaioni G, Marino MJ, Valenti O, Traynelis SF, Conn PJ (2001) Metabotropic glutamate receptors 1 and 5 differentially regulate CA1 pyramidal cell function. J Neurosci 21:5925-5934.

Moroz LL, Park JH, Winlow W (1993) Nitric oxide activates buccal motor patterns in Lymnaea stagnalis. Neuroreport 4:643-646.

Onodera M, Morita, Mano Y, Murota S (2000) Differential effects of nitric oxide on the activity of prostaglandin endoperoxide $\mathrm{H}$ synthase- 1 and -2 in vascular endothelial cells. Prostaglandins Leukot Essent Fatty Acids 62:161-167.

Otsuka E, Hirano K, Matsushita S, Inoue A, Hirose S, Yamaguchi A, Hagiwara H (1998) Effects of nitric oxide from exogenous nitric oxide donors on osteoblastic metabolism. Eur J Pharmacol 349:345-350.

Pedarzani P, Stocker M (2008) Molecular and cellular basis of small-and intermediate-conductance, calcium-activated potassium channel function in the brain. Cell Mol Life Sci 65:3196-3217.

Philippides A, Ott SR, Husbands P, Lovick TA, O'Shea M (2005) Modeling cooperative volume signaling in a plexus of nitric-oxide-synthaseexpressing neurons. J Neurosci 25:6520-6532.

Sah P, Faber ES (2002) Channels underlying neuronal calcium-activated potassium currents. Prog Neurobiol 66:345-353.

Schrofner S, Zsombok A, Hermann A, Kerschbaum HH (2004) Nitric oxide decreases a calcium-activated potassium current via activation of phosphodiesterase 2 in Helix U-cells. Brain Res 999:98-105.

Selverston A, Elson R, Rabinovich M, Huerta R, Abarbanel H (1998) Basic principles for generating motor output in the stomatogastric ganglion. Ann N Y Acad Sci 860:35-50.

Shao LR, Halvorsrud R, Borg-Graham L, Storm JF (1999) The role of BKtype $\mathrm{Ca}^{2+}$-dependent $\mathrm{K}^{+}$channels in spike broadening during repetitive firing in rat hippocampal pyramidal cells. J Physiol 521:135-146.

Sjöström PJ, Rancz EA, Roth A, Häusser M (2008) Dendritic excitability and synaptic plasticity. Physiol Rev 88:769-840.

Spencer GE, Lukowiak K, Syed NI (2000) Transmitter-receptor interactions between growth cones of identified Lymnaea neurons determine target cell selection in vitro. J Neurosci 20:8077-8086.

Spitzer NC (2006) Electrical activity in early neuronal development. Nature 444:707-712. 
Spitzer NC, Lautermilch NJ, Smith RD, Gomez TM (2000) Coding of neuronal differentiation by calcium transients. Bioessays 22:811-817.

Staras K, Gyóri J, Kemenes G (2002) Voltage-gated ionic currents in an identified modulatory cell type controlling molluscan feeding. Eur J Neurosci 15:109-119.

Steinert JR, Kopp-Scheinpflug C, Baker C, Challiss RA, Mistry R, Haustein MD, Griffin SJ, Tong H, Graham BP, Forsythe ID (2008) Nitric oxide is a volume transmitter regulating postsynaptic excitability at a glutamatergic synapse. Neuron 60:642-656.

Straub VA, Grant J, O’Shea M, Benjamin PR (2007) Modulation of serotonergic neurotransmission by nitric oxide. J Neurophysiol 97:1088-1099.

Tornieri K, Rehder V (2007) Nitric oxide release from a single cell affects filopodial motility on growth cones of neighboring neurons. Dev Neurobiol 67:1932-1943.

Trimm KR, Rehder V (2004) Nitric oxide acts as a slow-down and search signal in developing neurites. Eur J Neurosci 19:809-818.

Van Wagenen S, Rehder V (1999) Regulation of neuronal growth cone filopodia by nitric oxide. J Neurobiol 39:168-185.

Van Wagenen S, Rehder V (2001) Regulation of neuronal growth cone filopodia by nitric oxide depends on soluble guanylyl cyclase. J Neurobiol 46:206-219.

Van Wagenen S, Cheng S, Rehder V (1999) Stimulation-induced changes in filopodial dynamics determine the action radius of growth cones in the snail Helisoma trivolvis. Cell Motil Cytoskeleton 44:248-262.
Vatanparast J, Janahmadi M (2009) Contribution of apamin-sensitive SK channels to the firing precision but not to the slow afterhyperpolarization and spike frequency adaptation in snail neurons. Brain Res 1255:57-66.

Welshhans K, Rehder V (2005) Local activation of the nitric oxide/cyclic guanosine monophosphate pathway in growth cones regulates filopodial length via protein kinase $\mathrm{G}$, cyclic ADP ribose and intracellular $\mathrm{Ca}^{2+}$ release. Eur J Neurosci 22:3006-3016.

Welshhans K, Rehder V (2007) Nitric oxide regulates growth cone filopodial dynamics via ryanodine receptor-mediated calcium release. Eur J Neurosci 26:1537-1547.

Wood J, Garthwaite J (1994) Models of the diffusional spread of nitric oxide: implications for neural nitric oxide signalling and its pharmacological properties. Neuropharmacology 33:1235-1244.

Yang S, Cox CL (2008) Excitatory and anti-oscillatory actions of nitric oxide in thalamus. J Physiol 586:3617-3628.

Young SR, Bianchi R, Wong RK (2008) Signaling mechanisms underlying group I mGluR-induced persistent AHP suppression in CA3 hippocampal neurons. J Neurophysiol 99:1105-1118.

Zhuo M, Hawkins RD (1995) Long-term depression: a learning-related type of synaptic plasticity in the mammalian central nervous system. Rev Neurosci 6:259-277.

Ziff EB (2007) TARPs and the AMPA receptor trafficking paradox. Neuron 53:627-633. 